\section{$i^{8}$ \\ LA-7436-MS}

Informal Report

\section{Water Supply at Los Alamos During 1977}

$\frac{\pi}{\frac{5}{5}}$

to

$\frac{7}{\omega}$

$\Phi$

$\stackrel{ \pm}{5}$ 
LA-7436-MS

Informal Report

UC-11

Issued: August 1978

\title{
Water Supply at Los Alamos During 1977
}

\author{
William D. Purtymun
}




\section{WATER SLPPIY A'T LOS ALAMOS DLRING; 1977 \\ hy}

William I). Purtymun

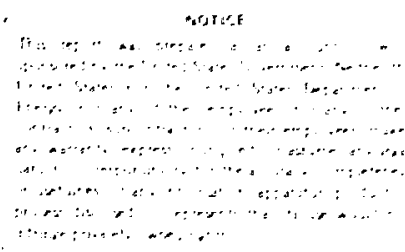

ABST'TRACT

The Los Alamos water supply for 1977 consisted of $1.17 .4 \times 10^{k}$ gal from wells in three fields and $57 \times 10^{8}$ gal from the gallery in Water (Canyon. The production from the well fields was at its lowest volume since 1971). Waterlevel trends were as anticipated under current production practices. Well rehabilitation should be continued to ensure an adequate and reliable supply from wells that are 10 to over 25 yr old.

\section{INTRODUCTION}

This report summarizes pumpage and atpuifer conditions for wells in the los Alamos, Ciuaje, and Pajarito well lields (Fig. 1). These wells supply most of the water used for municipal and industrial purposes in Los Alamos. The gallery in Water ('anyon that supplies the balance to the system is also dis. cussed. This report is a joint effort between (iroup H-8 of the Los Alamos Scientific l aboratory (LASL) and the Utilities and Engineering Division of the Zia Company (Zia U/E). Its purpose is to ensure a continuing historical record and to provide guidance for management of water resources and long-range planning for the water supply system. One summary report and six annual reports have been issued as the result of these studies. ${ }^{1-7}$ The eighth report extrapolates water-level trends in the well fields to 1983 under current production. $^{8}$

The Zia U/E, the Department of Energy (DOE) support contractor at Los Alamos, maintains and operates the water supply system. Water is pumped from weils, through transmission lines, and lifted by booster pumps into reservoirs for storage and distribution to the community and Laboratory areas
(Fig. 1). Water from the gallery flows by gravity through a microfilter station and is pumped intorome of the system reservoirs for distribution. Zia $/ / F$. maintains monthly records of hours of operation on each well, along with daily and monthly production records. Monthly average nonpumping and pumping water levels are computed from air-line pressure data recorded continuously at each well. Thete data provide input for calculating pumping rates. drawdown (difference between nonpumping and pumping water levels). specific capacity (punp rate per unit drawdown), and other well-field statistics in. cluded in this report.

Hydrographs have been prepared for one observation well, one standby supply well. 15 supply wells. and the gallery in Water Canyon. The hydrographs for the wells show annual average nonpumping and pumping water levels. specific capacity, and annual pumpage for the years during which the wells have been in production. The hydrograph for the gallery presents annual production and the annual average discharge rate. Appendixes $\mathrm{A}$ and $\mathrm{B}$ contain basic pumping and production information for each supply well, monthly for 1977 and annually for the period of record. 


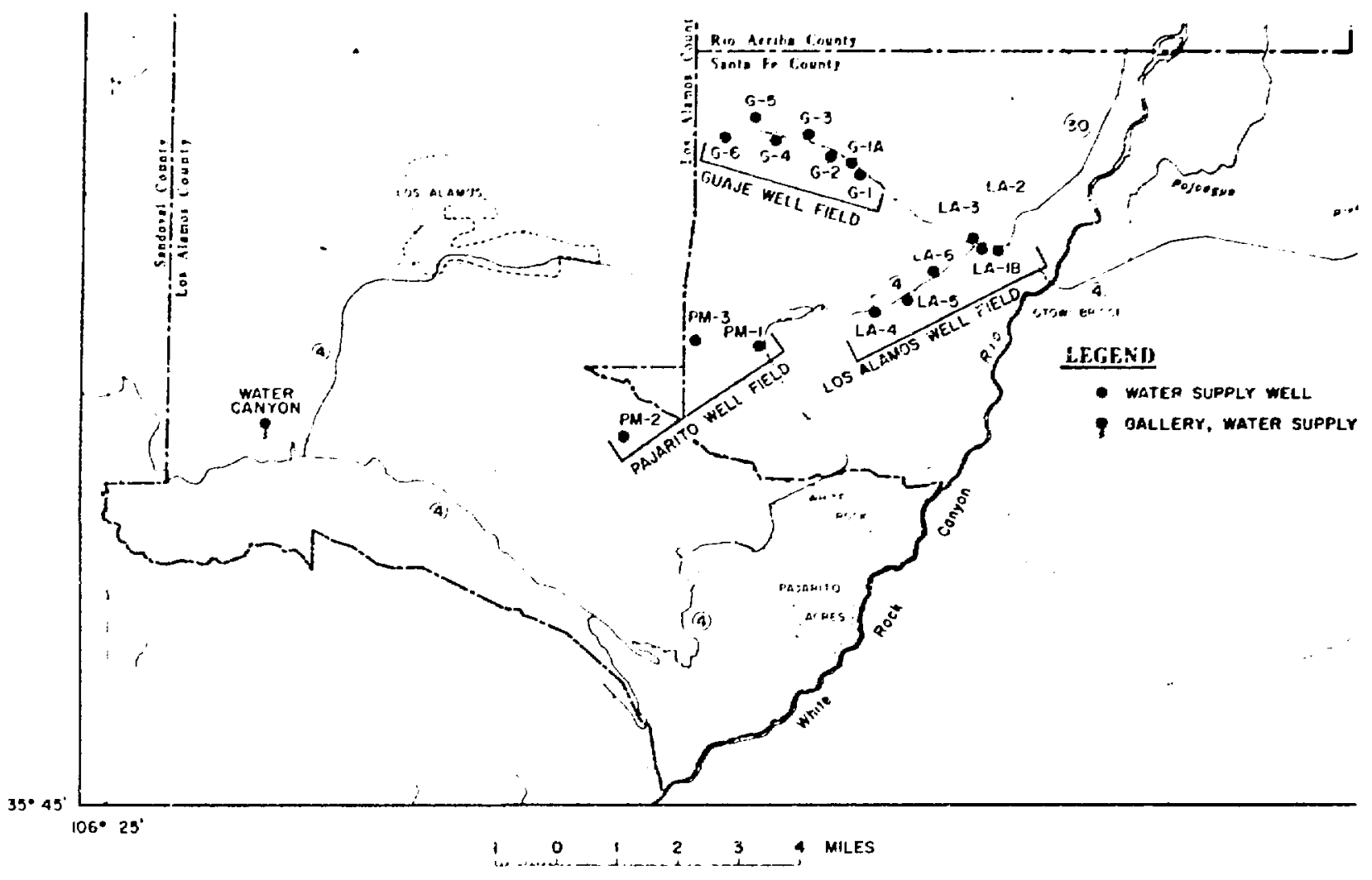

Fig. 1.

Location of well fields, supply wells, and gallery water supply.

\section{WELL-FIELD CHARAC'TERISTICS}

Production from the three weli fields decreased $217 \times 10^{8}$ gal from $1691 \times 10^{8}$ gal in 1976 to $1474 \times$ $10^{8} \mathrm{gal}$ in 1977 ('Table 1). The production declined to its lowest point since 1970 in spite of a larger number of residents, as represented by the completion of 140 housing units in Los Alamos and 117 units in White Rock. This decline was partly because of early summer rains, which reduced demand for lawn irrigation, and partly because of conservation by residents of Los Alamos County, possibly encouraged by increased water rates. The decline was accompanied by a relative shift in area of demand. Production into Los Alamos from the Los Alamos and Guaje Fields decreased only $14 \times 10^{\circ}$ gal, whereas that in White Rock from the Pajarito Field decreased $203 \times$ $10^{\circ} \mathrm{gal}$. Water production in 1977 fell below the projected demand by $620 \times 10^{\circ}$ gal (Fig. 2). ${ }^{\circ}$
The peak demand period in 1977 was for the 19 day period of May 31 through .June 18 when pumpage was about $149 \times 10^{\circ}$ gal, or $7.8 \times 10^{\circ} \mathrm{gal} / \mathrm{day}$. Voluntary reduction of water use by the residents ended the peak demand period during the La Mesa fire, in order to maintain maximum storage for lire protection. The production decreased $4 \times 10^{6} \mathrm{kal} /$ day when the emergency was announced. Daily production during the 19 day period exceeded $10 \times$ $10^{6}$ gal for 2 days, $9 \times 10^{8} \mathrm{gal}$ for 4 days, and $8 \times 10^{6}$ gal for 4 days. During the remaining 9 days, production was less than $8 \times 10^{\circ} \mathrm{gal} / \mathrm{day}$. In comparison. the peak demand period in 1976 was for 32 days when production was $299 \times 10^{\circ} \mathrm{gal}$ for an average of $9.4 \times 10^{\circ} \mathrm{gal} / \mathrm{day}$. During this period, the production exceeded $10 \times 10^{6} \mathrm{gal} /$ day for 14 days, $9 \times 10^{6}$ gal/day for 9 days, and $8 \times 10^{8}$ gal/day for 4 days. During the remaining 5 days, production was less than $8 \times 10^{8} \mathrm{gal} /$ day. 
TABLE I

PRODC CTION IN MILLIONS OF GALLGNS FROM WELLS AND GALLERY

$19.17 \quad 1978$

\begin{tabular}{|c|c|c|c|c|c|}
\hline Year & $\begin{array}{l}\text { Los Alamos } \\
\text { Field }\end{array}$ & $\begin{array}{l}\text { Guaje } \\
\text { Field }\end{array}$ & $\begin{array}{l}\text { Pajarito } \\
\text { Field }\end{array}$ & $\begin{array}{c}\text { Water Canyon } \\
\text { Gallery }\end{array}$ & $\begin{array}{l}\text { Production } \\
\text { Total }\end{array}$ \\
\hline 1947 & 1.47 & 0 & 0 & 8.4 & 231 \\
\hline $19+4 x$ & $26 \sqrt{3}-2$ & (1) & 0 & 97 & 361 \\
\hline 194.4 & 3110 & 0 & 0 & 9.2 & 394 \\
\hline$\{1 ; \div 1\}$ & $\therefore 4 \pi$ & 3 & 0 & 54 & 601.4 \\
\hline $195: 1$ & 712 & 638 & () & 39 & $x(0,4$ \\
\hline 1952 & $44 x$ & 3501 & 0 & 48 & $x .46$ \\
\hline 195.73 & 4.44 & 372 & () & 39 & kisit \\
\hline 195.4 & 380 & 37.4 & (1) & 40) & 79.4 \\
\hline $19 \overline{15}$ & 4117 & 375 & () & 33 & 81.5 \\
\hline $19: \overline{6 i}$ & 437 & 506 & () & $2: 3$ & 9665 \\
\hline $195 \overline{1}$ & $3.51)$ & 378 & () & 4) & 768 \\
\hline $19 \overline{1} \times$ & $: 372$ & 395 & () & 60) & 827 \\
\hline $195 \%$ & 391 & 478 & 0 & 54 & $92: 3$ \\
\hline 196601 & 530 & 5333 & 0 & 48 & 1111 \\
\hline 19661 & 546 & 624 & 0 & 54 & 1224 \\
\hline $196 i_{2}$ & דה & 597 & (1) & 67 & 1241 \\
\hline $196: 3$ & 539 & 654 & 0 & 51 & 1244 \\
\hline 1964 & 627 & $66 \overline{5}$ & 0 & 4.5 & 1337 \\
\hline 1965 & 447 & 571 & 99 & 72 & 1189 \\
\hline 1966 & 450 & 613 & 127 & 82 & 1272 \\
\hline 1967 & 373 & 464 & 481 & 56 & 1374 \\
\hline 1968 & 34.5 & 474 & 584 & 65 & 1468 \\
\hline 1969 & $33.3 \mathrm{i}$ & 435 & 569 & 80 & 1415 \\
\hline 1970 & 360 & 423 & 595 & 65 & 1443 \\
\hline 1971 & 412 & 484 & 657 & 37 & 1590 \\
\hline 1972 & 380 & 467 & 662 & 40 & 1549 \\
\hline 1973 & 406 & 475 & 685 & 49 & 1615 \\
\hline 1974 & 369 & 453 & 802 & 35 & 1659 \\
\hline 1975 & 356 & 4.31 & 749 & 42 & 1578 \\
\hline 1976 & 34.3 & 5.31 & 817 & 41 & 1732 \\
\hline$\underline{1977}$ & 34.5 & 515 & 614 & $5 \pi$ & 1531 \\
\hline Total & 12927 & 12708 & 7441 & 1689 & 34765 \\
\hline
\end{tabular}




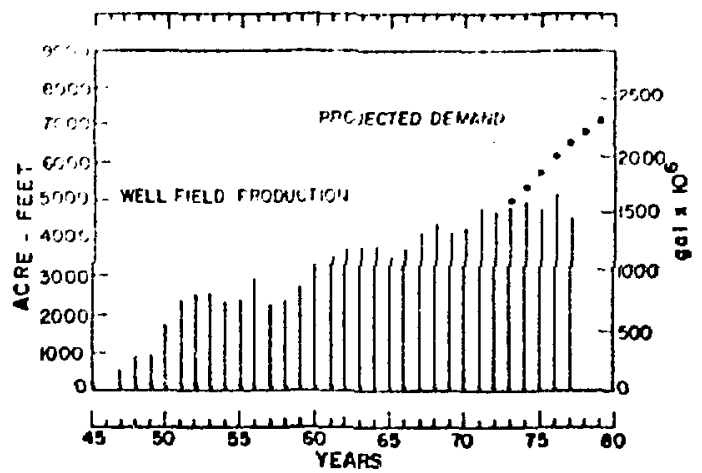

Fig. 2

Well-field production 1947-77 and projected demand 197:3-79.

The heaviest demand for water in 1959 was in Vay, fune, and July, when about $578 \times 10^{8}$ gal were used, representing about $38 \%$ of the total production of 1977. The months of least demand were December, fanuary, and february, when production was 260()$\times 10^{8}$ gal, representing about $17 \%$ of the total production. Winter leve's in the wolls fluctuated with pumpage. The highest water levels were during the months of least pumpage (December, January, and February, whereas lowest water levels were during months of greatest pumpage (May, Jume, and July).

Total production from the well fields and gallery since 1947 has been $34765 \times 10^{8}$ yal. Of this, the wells have produced $333076 \times 10^{6}$ gal, or $95 \%$ (Table I). The annual production, per cent pumpage by field, and per cent of pumpage of individual wells are given in Table II. The average annual pumping rate for individual wells for the period 1973 through 1977 is shown in Table III.

\section{A. Los Alamos Field}

The Los Alamos well field is composed of six supply wells and one observation well. The production in 1977 was from five supply wells; the sixth is on standby status for emergency use only. ${ }^{10}$ Pumpage from the field increased by $2 \times 10^{\circ} \mathrm{gal}$, from $343 \times$ $10^{\circ} \mathrm{gal}$ in 1976 to $345 \times 10^{\circ} \mathrm{gal}$ in 1977 . The field produced about $22 \%$ of the total for the year (Table II).
Pumpage from the individual wells increated only - lightly above the 1976 productions. The water levein wells IA-1. IAA-1H. IA-2. IA-3. IA-A. and LA-:) varied slightly, but did not change significantly Ifigs. is through 8 . respectively.

Well LA-fi. on standby status. was pumped only three times during the year for $16-\mathrm{sing}$ and maintenance of the pump. About $1.7 \times 11^{p}$ gal of water was pumped to waste during the year. The restricted use of the well has resulted in higher water levels in 1977 than in 1975 or 1976 ifig. 91. The waler levels in $\mathrm{LA}$-f $\mathrm{f}$ rose about $14 \mathrm{ft}$ during the vear. whereas in the upper portion of the field $1 / A-4$ and -5) there was no significant change (Fig. 1). In the lower part of the field $(1, A-1,-1 B .-2$. and $\therefore$. the average water level decline was about 2 tt.

The average annual purapmy rate trom the tive producing wells in ine field declined about 27 gpm from $2124 \mathrm{gpm}$ in $1976 \mathrm{10} 20 \mathrm{~g} 7 \mathrm{gpm}$ in 197. The largest decline occurred at thell IA-1B ITable Ifl. It appears that the meter may have been reading : $\mathrm{t}$ " $5 \%$ low, which would account for the decreased pumping rate. It this is so, production for the sear would be about $\& \times 11^{s}$ gal greater than reported. The specific capacities of the five producing wells also showed no significant change in 197.7 fom 1976 data.

\section{B. Guaje Well-lield}

The Guaje well field is composed of seven wells (Fig. 1). The pumpage from the field decreased $16 \times$ $10^{6} \mathrm{gal}$ from $531 \times 10^{8} \mathrm{gal}$ in $197610515 \times 10^{8} \mathrm{gal}$ in 1977 (Table 1). The field produced $34^{\circ} \circ$ of the toral production (Table II). The pumpage declined in all wells except $\mathrm{G}-4$ where pumpage increased $15 \times 10^{6}$ gal above the pumpage of 1976 .

There was no significant change in annual average nonpumping levels in wells G-1, -1A. -2, $-3,-4,-\overline{3}$. and -6 (Figs. 10 through 16, respectively) when compared to 1976 water levels. The pumping level in well G-1 shows a continuing decline accompanied by a continuing decrease in specific capacity (Fig. 10). Rehabilitation of the pump and weli was performed in early $197 \%$. The intake of the pump was set $20 \mathrm{ft}$ higher at $500 \mathrm{ft}$, about $4 \mathrm{ft}$ above the reduction of the casing from 12 to $10 \mathrm{in}$., to allow free intake of water to the pump. Cleaning the casing and removal of 


\section{TABLE II}

\section{PRODCCTION PERCENTAGES}

1977

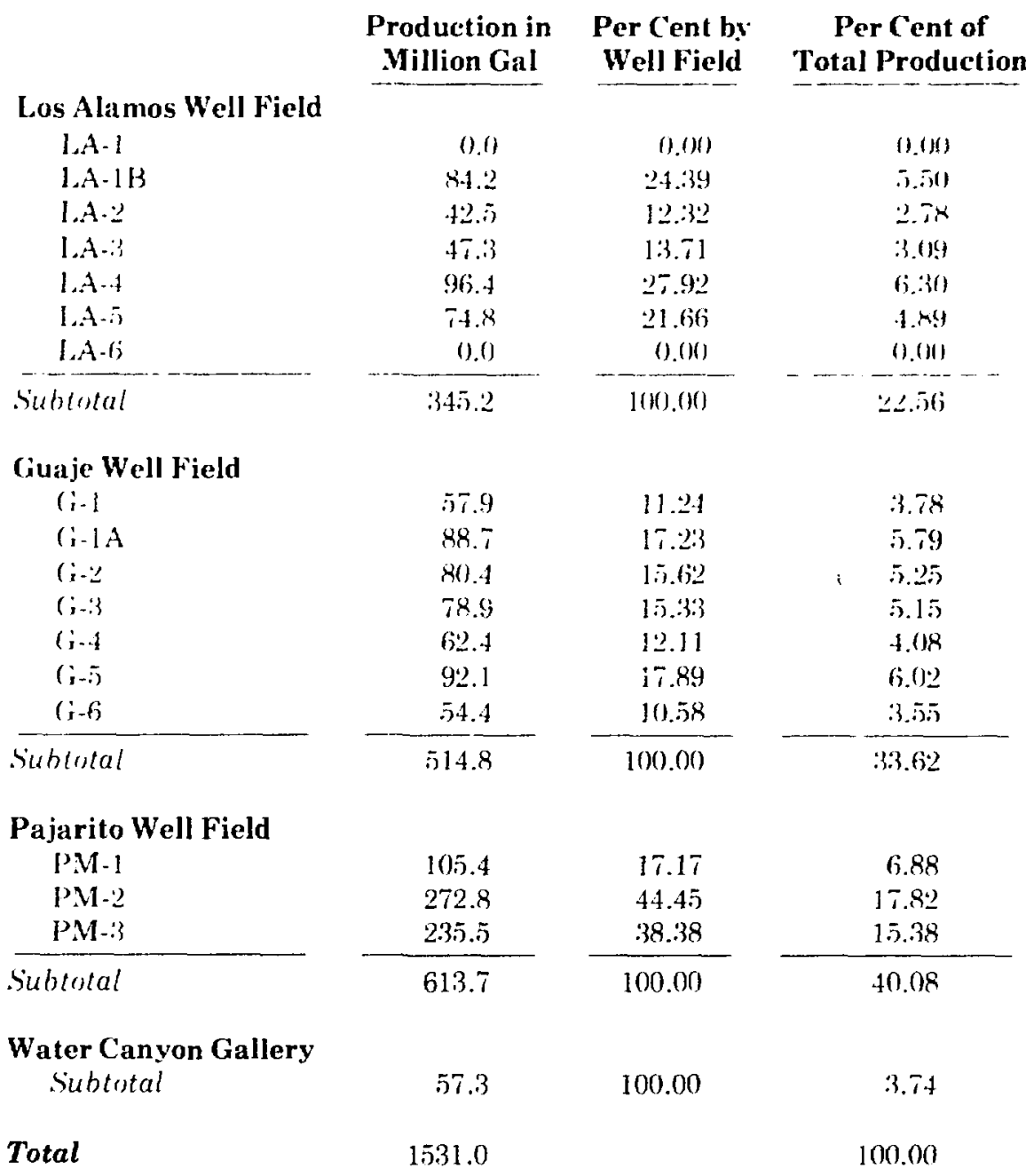

sediments from the well did not improve the specific capacity. The decline in the nonpumping water level and specific capacity results from interference by the nearby well G-1A.

The pumping level in well G-4 declined with increased pumpage, whereas the nonpumping level remained about the same (Fig. 14). As the pumping rate was decreased, the specific capacity did not change significantly.

The average annual pumping rate from the field decreased about $42 \mathrm{gpm}$ from $2991 \mathrm{gpm}$ in $19 \% 6$ to
$2949 \mathrm{gpm}$ in 1977 . The rates from individual wells during 1977 were only slightly different than in 1976 (Table III). The pumping rate of well $G-6$ has been reduced to keep the pumping level above the top of the pump. The specific capacity of the well has remained about the same: howerer. inspection of sand samples indicates some metal. line shaft, or pump wear. 
TABLE III

\section{AVERAGE ANNUAL PUMPING RATE OF WELLS AND DISCHARGE FROM GALLERY \\ $1973-1977$ \\ (in $\mathrm{gpm}$ )}

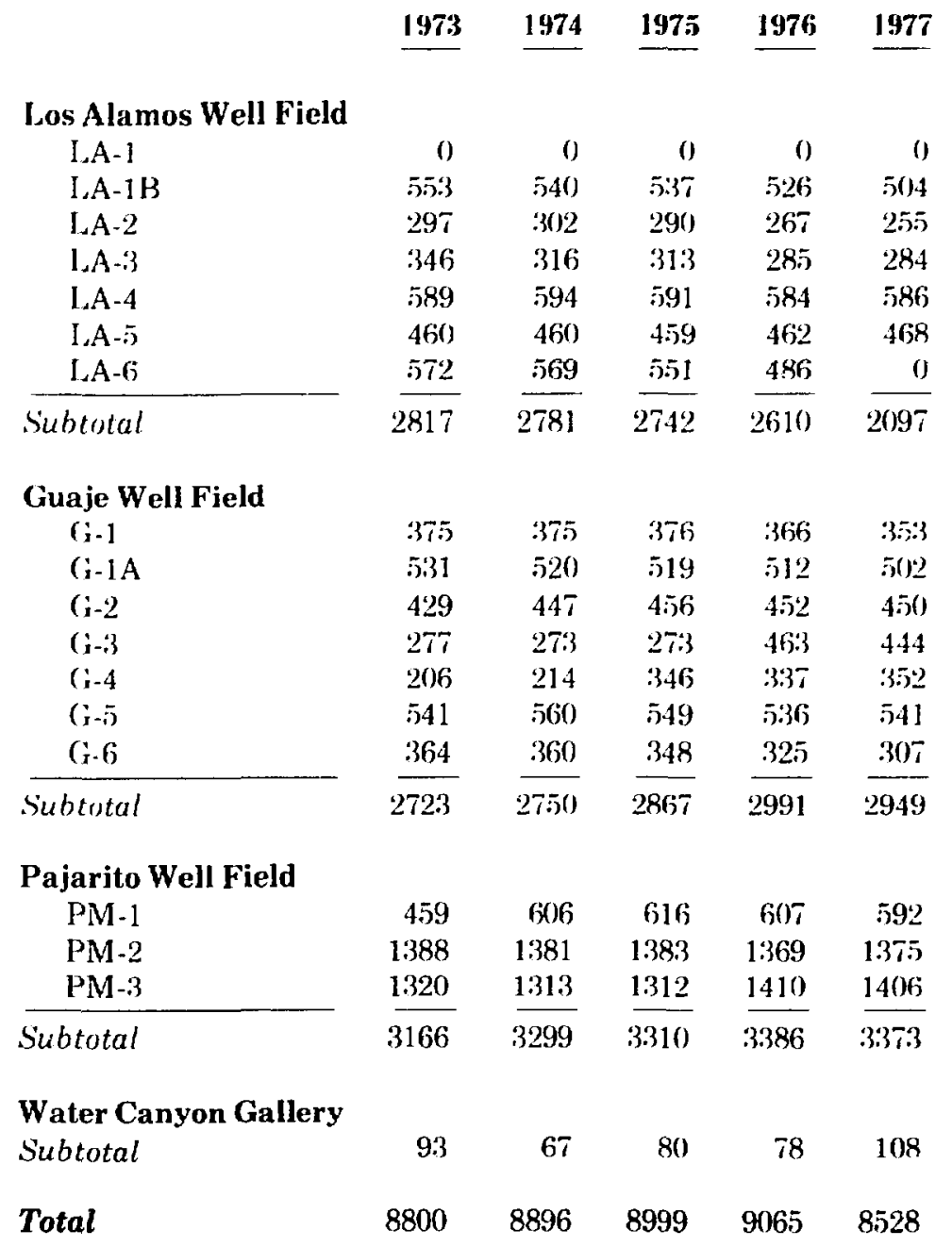

\section{Pajarito Well Field}

The Pajarito well field is composed of three wells (Fig. 1). The pumpage from the wells decreased 203 $\times 10^{\circ}$ gal from $817 \times 10^{\circ}$ in 1976 to $614 \times 10^{\circ} \mathrm{gal}$ in 1977. The decreases in individual wells were $1.4 \times$ $10^{8}$ gal from PM-1, $169.2 \times 10^{\circ}$ gal from PM-2, and $32.8 \times 10^{\circ}$ gal from PM-3. The field produced $40 \%$ of total production (Table II).
Water levels remained about the same as in 1976 in wells PM-1, -2 , and -3 (Figs. 17 through 19 , respectively) in spite of the decrease in pumpage. The average annual pumping rate from the field declined about $13 \mathrm{gpm}$ from $3386 \mathrm{gpm}$ in 1976 to $3373 \mathrm{gpm}$ in 1977 . This change was not considered significant, as the largest decrease $(15 \mathrm{gpm})$ occurred at PM-1 and was the result of a change in back pressure in the transmission line when PM-3 was 

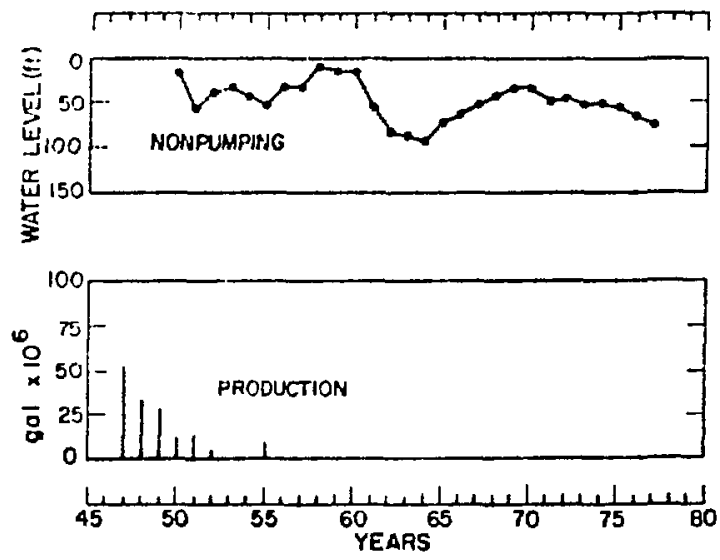

lig. 3 .

Anmual alvoras mompumping uater lecel and annual preductim. Las Alamos Hell l.A.l
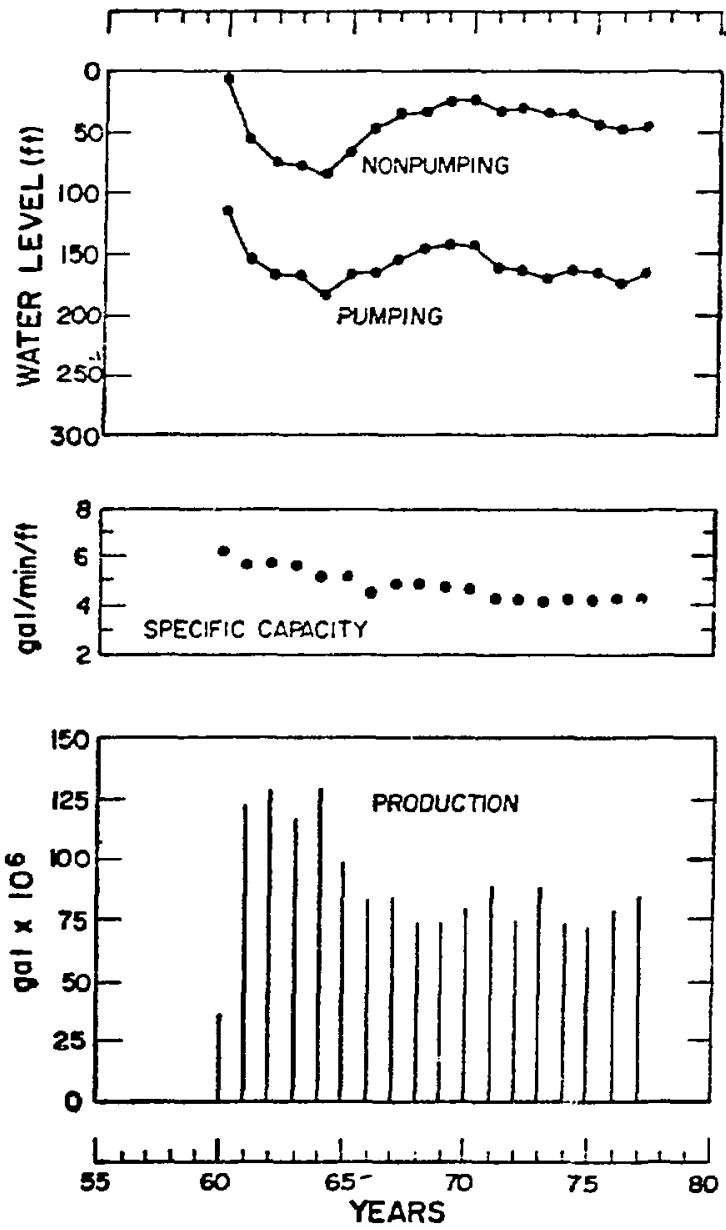

Fig. 4.

Annual average nonpumping and pumping uater levels. annual aberage specific capacity, and annual production, Los Alamos Well LA-IB.
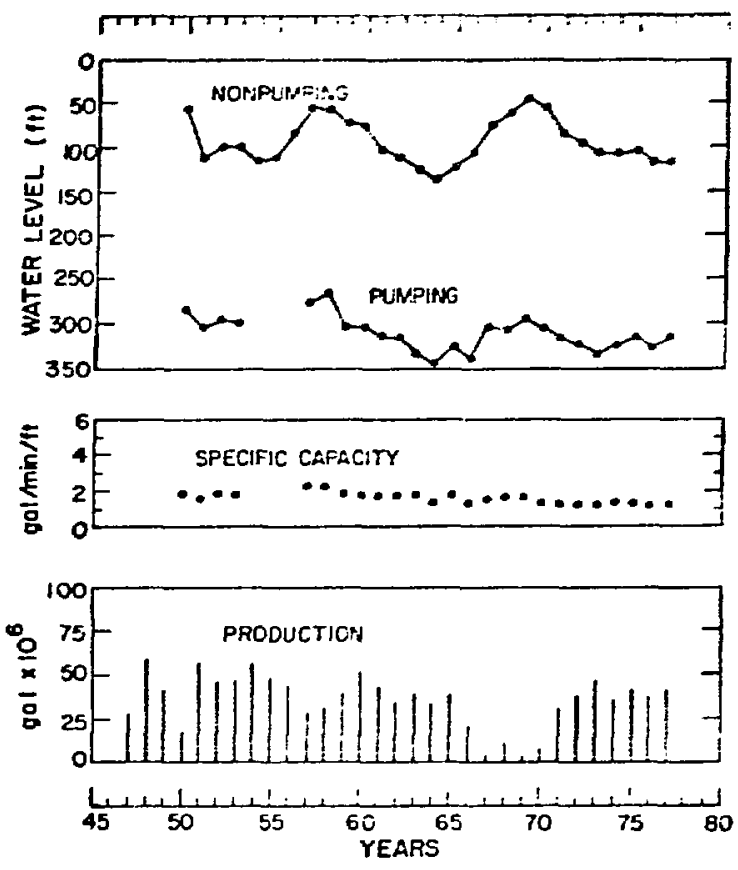

Fin. ;.

Annual a rork' nempumping and pumping uater levels. annual aurage sesecifac capacits, and annual production. Les Alamos biell LAL-2
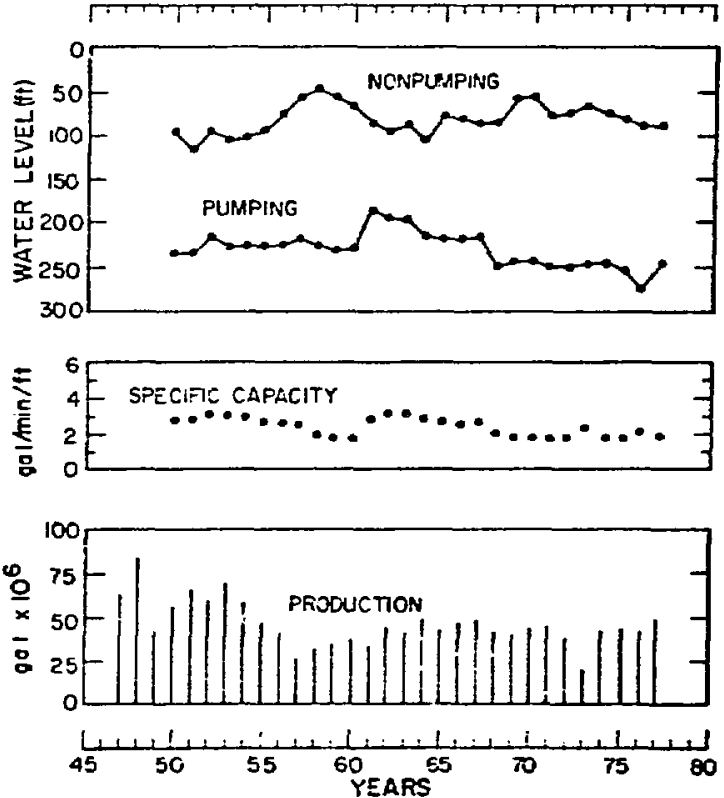

Fig. 6.

Annual acerage nonpumping and pumping uater lecels. ammual acerage specific capacit?, and anmual production. Los Alamos Well LA -3. 

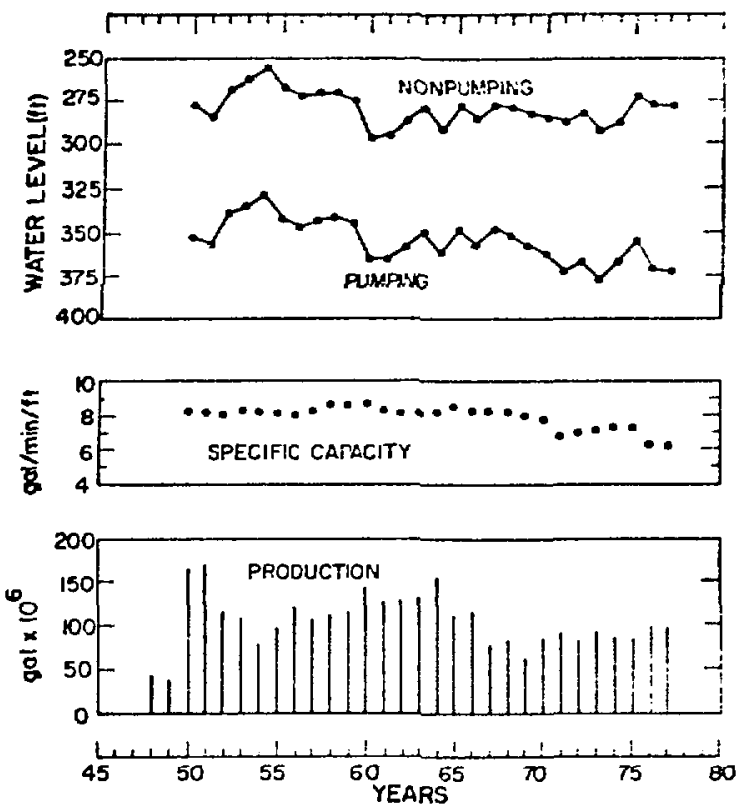

Figt. 7 .

Annual acerage nisnsumping and pumping uater levels. annual atcrase' specific capacity, and annual production. Los Alamos Woll LA-A.
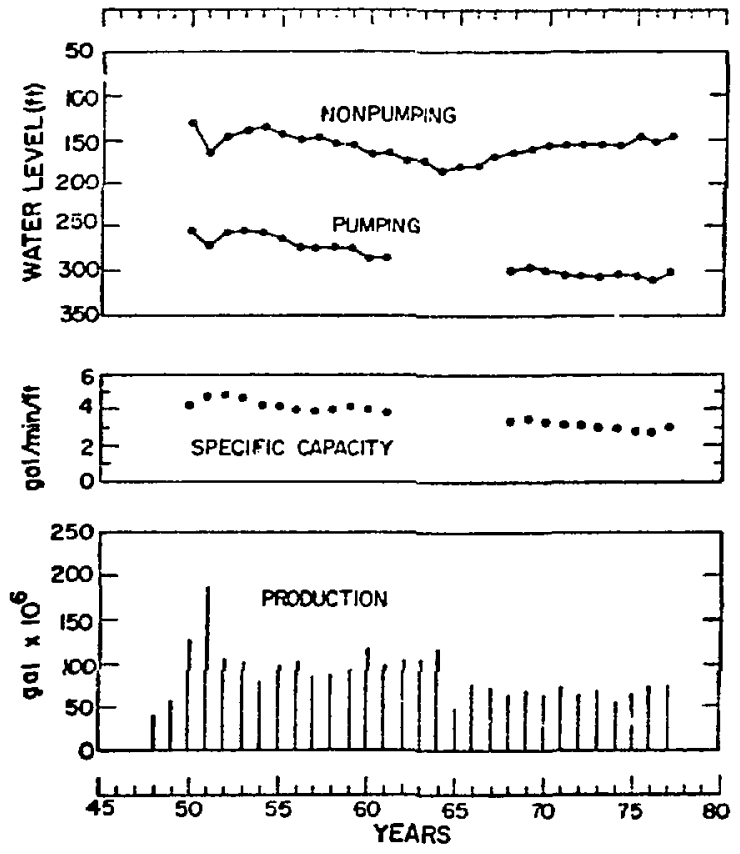

Fig. 8.

Annual average nonpumping and pumping water levels, annual average specific capacity, and annual production, Los Alamos Well LA -5.
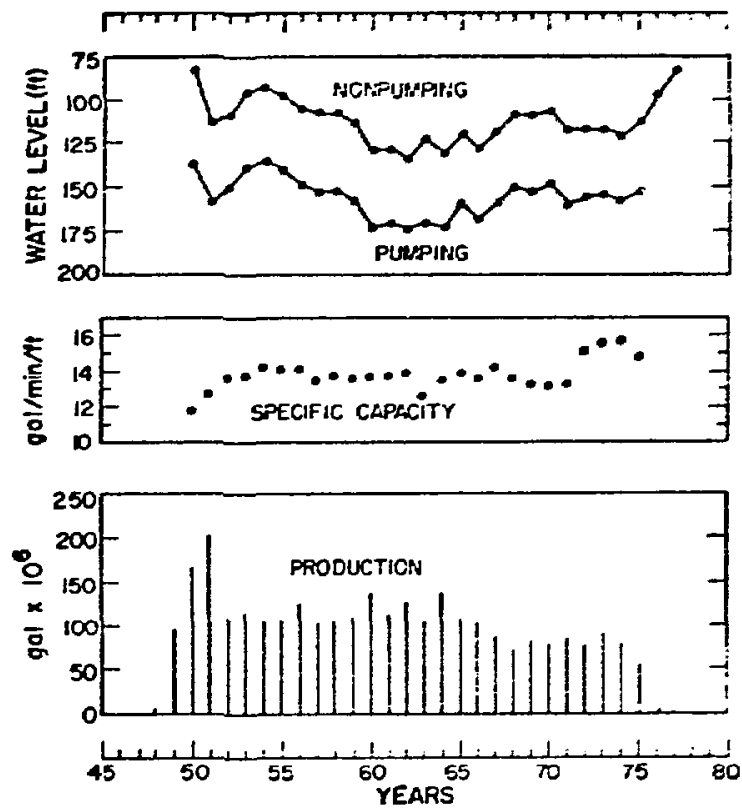

Fig. 9.

Annual atcrage nonpumping and pumping uater levels. annual acerage specific capacits, and annual production, Los Alamos Well LA-6.
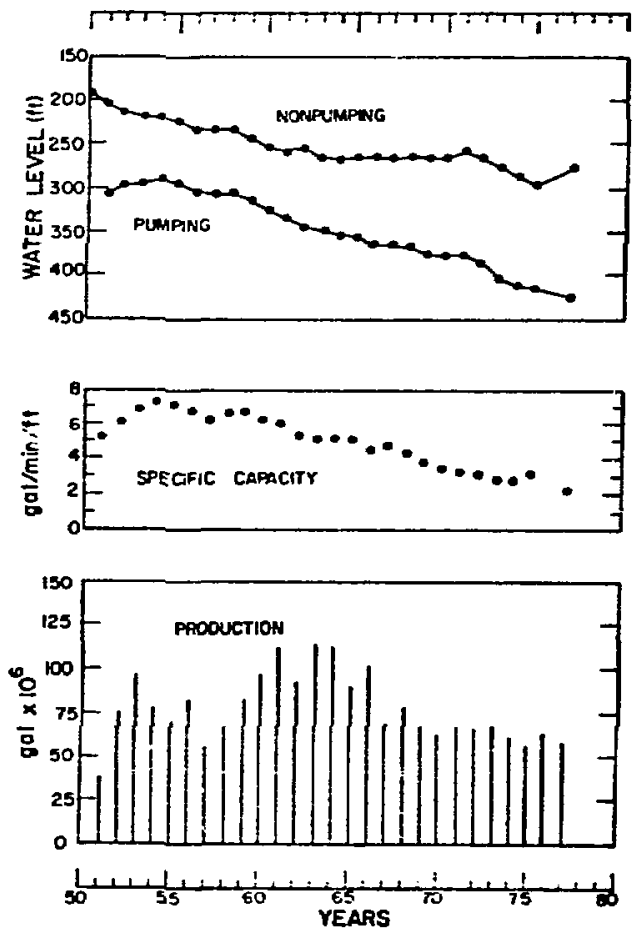

Fig. 10.

Anmual acerage nonpumping and pumping uater levels. annual acerage specific capacity, and annual production. Guaje Well (i-1. 

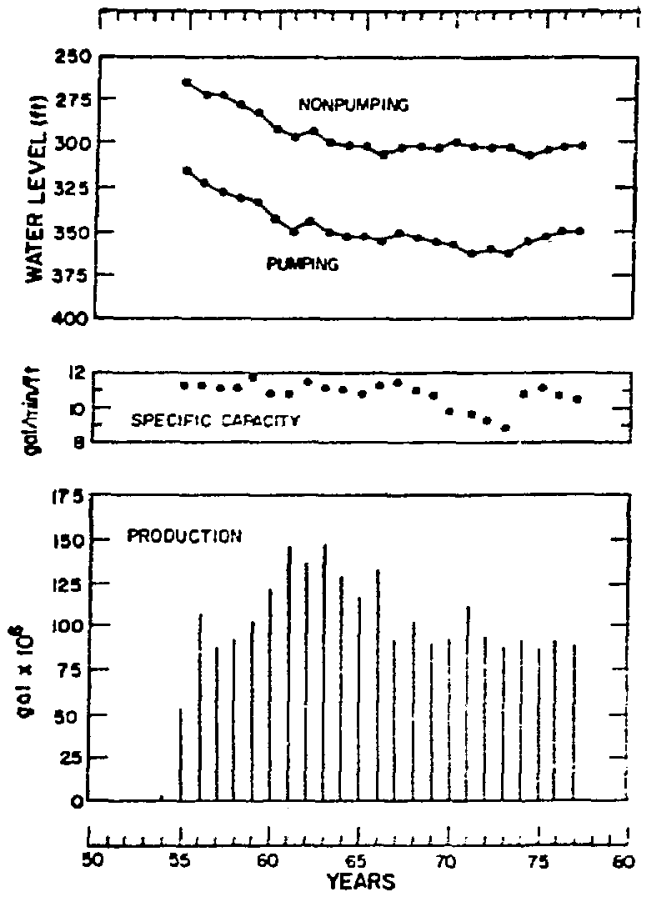

Fig. 11 .

Annual acerage numpumping and pumping water levels, annual acerage' specific capacity, and annual production. (iuaje Well G-1A.
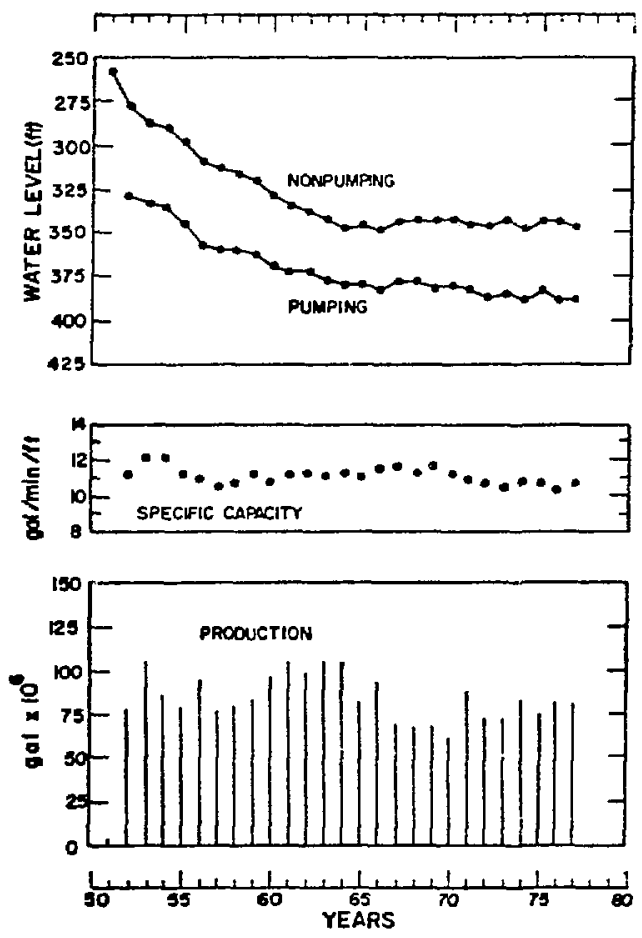

Fig. 12.

Annual average nonpumping and pumping uater levels. annual average specific capacity, and annual production, Guaje Well G-2.
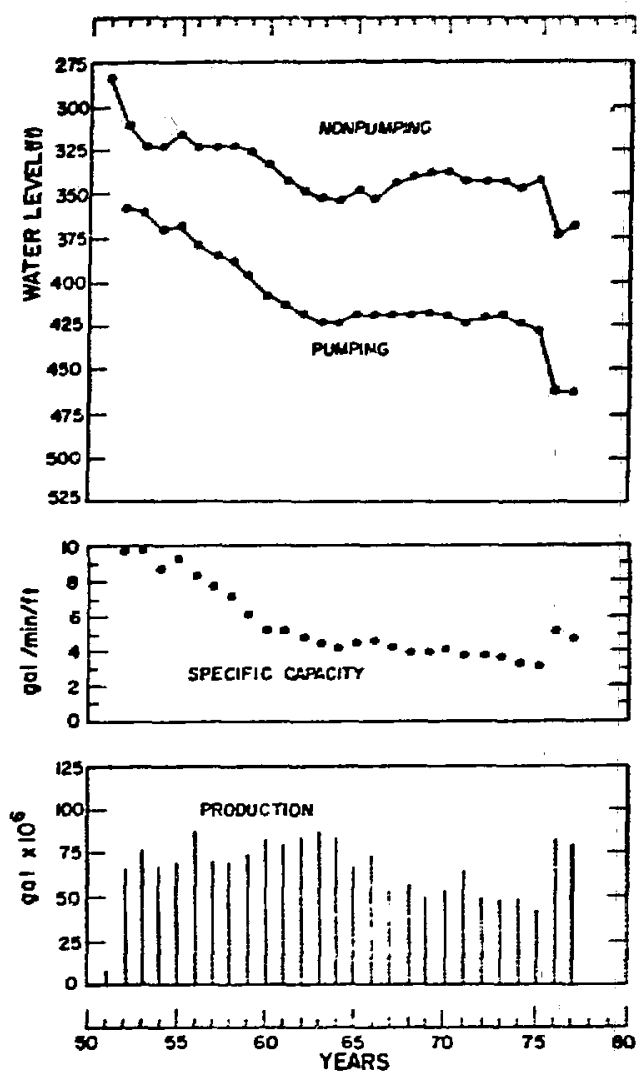

Fig. 13 .

Annual acerage nompumping and pumping uater lecels annual acerage specific capacit3, and anneal production. Guaje Well (i-3).
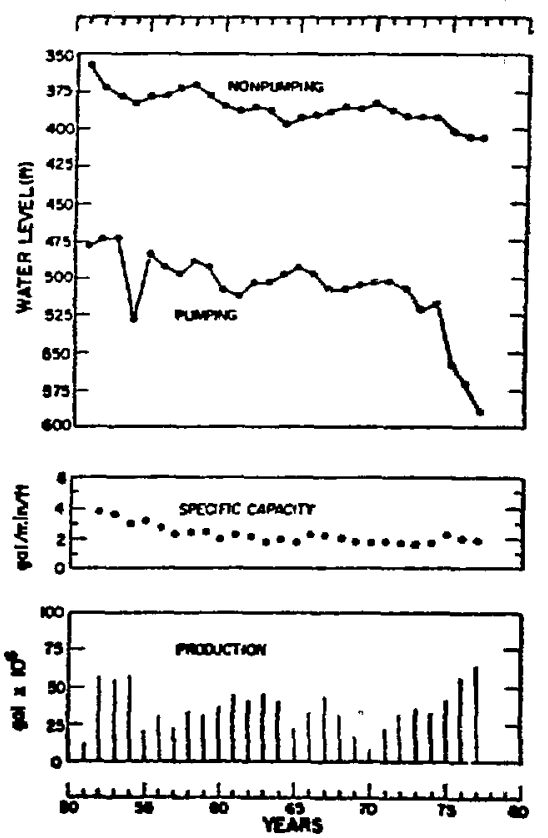

Fig. 14.

Annual acerage tonpumping and pumping uater letels. anmual auenage specific capacity, and anmual production. Guaje Well G-4. 

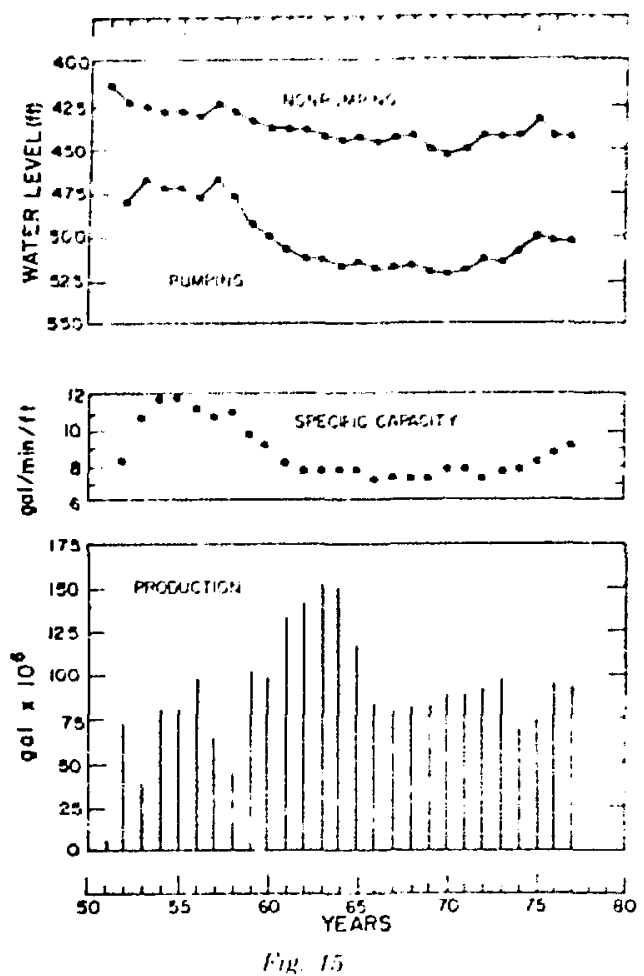

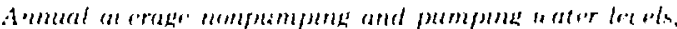

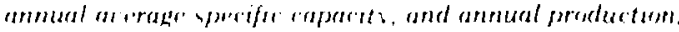
liulu, Hidl $(;-i)$

TTTTTTTT:TTT:
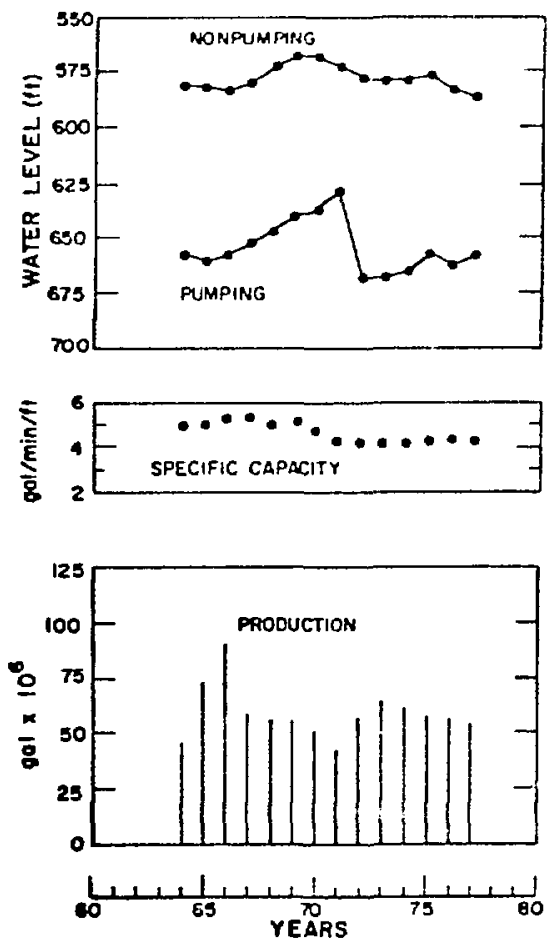

Fig. 16.

Annual average nonpumping and pumping water levels, annual acerage specific capacity, and annual production. Guaje Woll G-6.
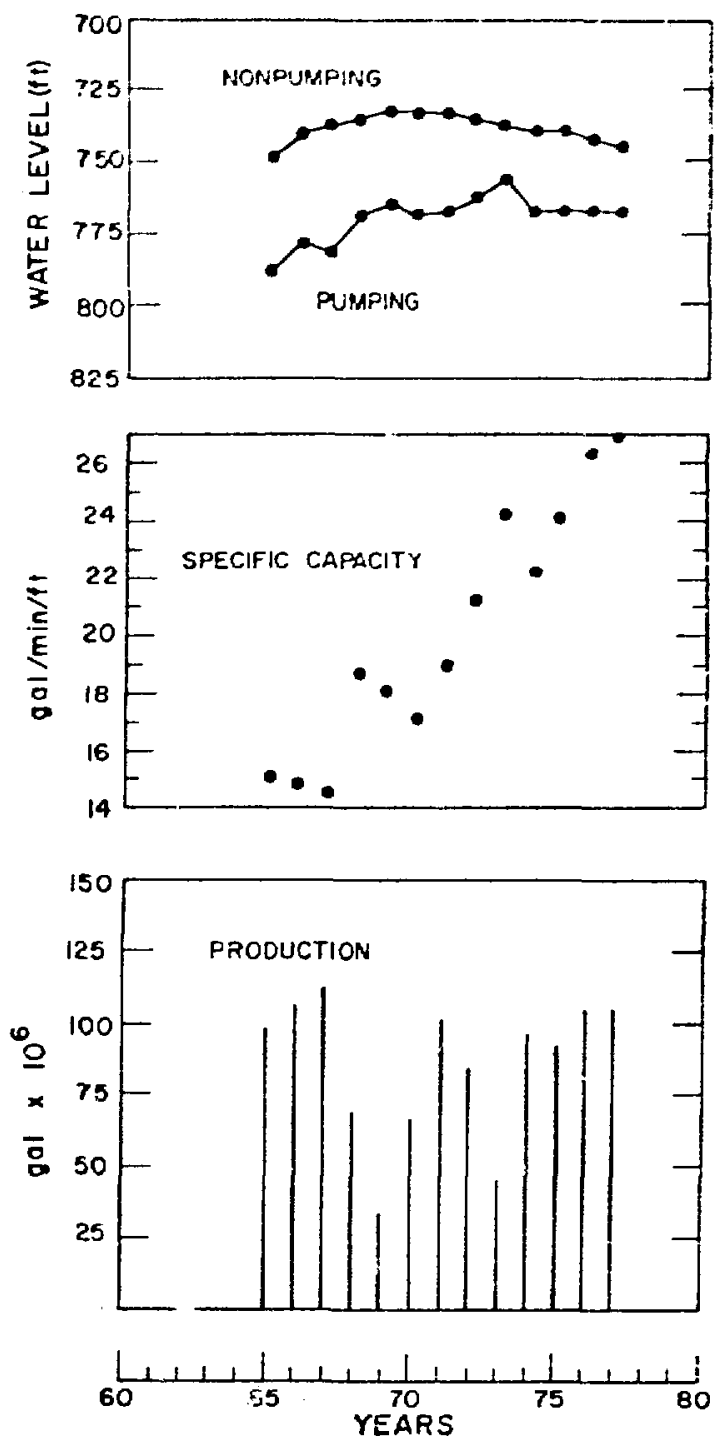

Fig. 17.

Ammal acerage nompumping and pumping uater lecels. annual acerage' specific capacits, and annual praduction. Pajarite Well PM.I. 

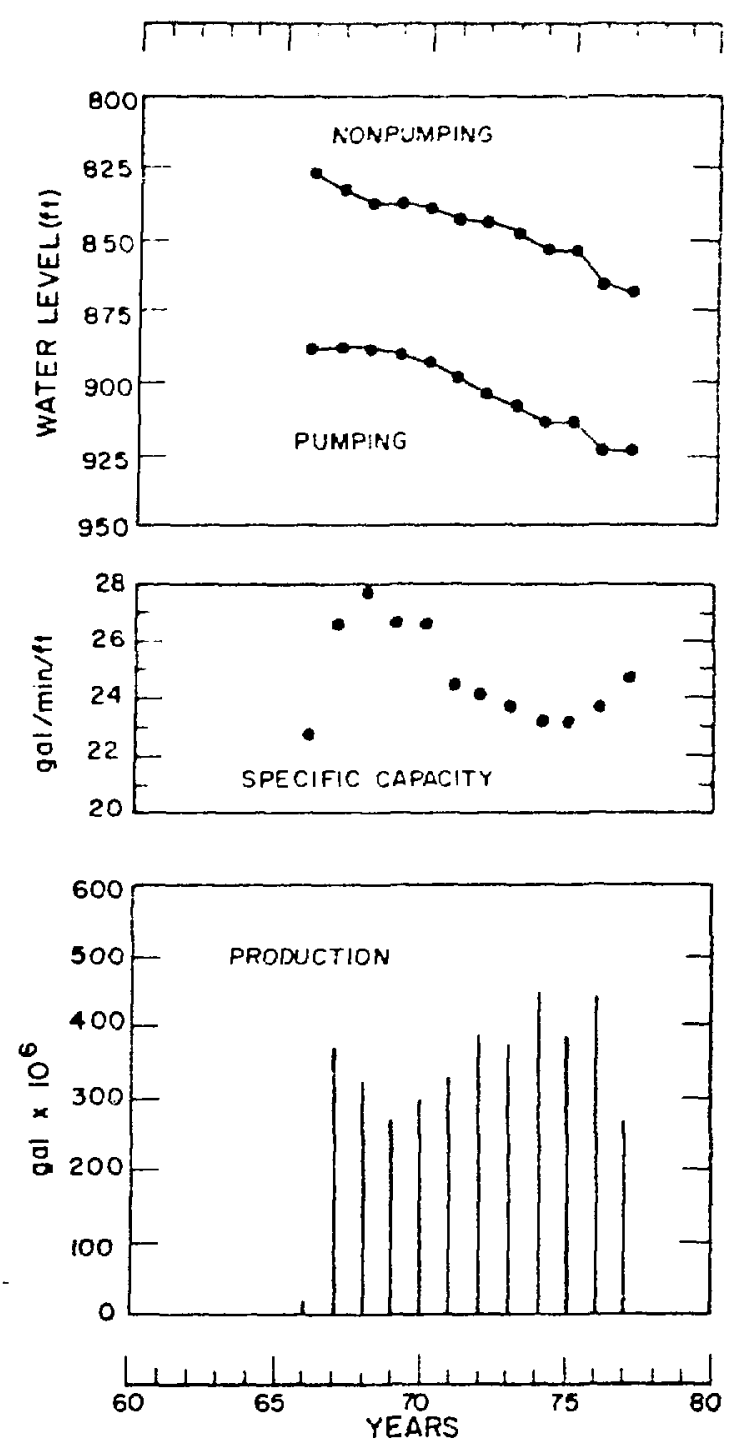

Fig. 18.

Annual average nonpumping and pumping water levels, annual average specific capacity, and annual production, Pajarito Well PM-2.
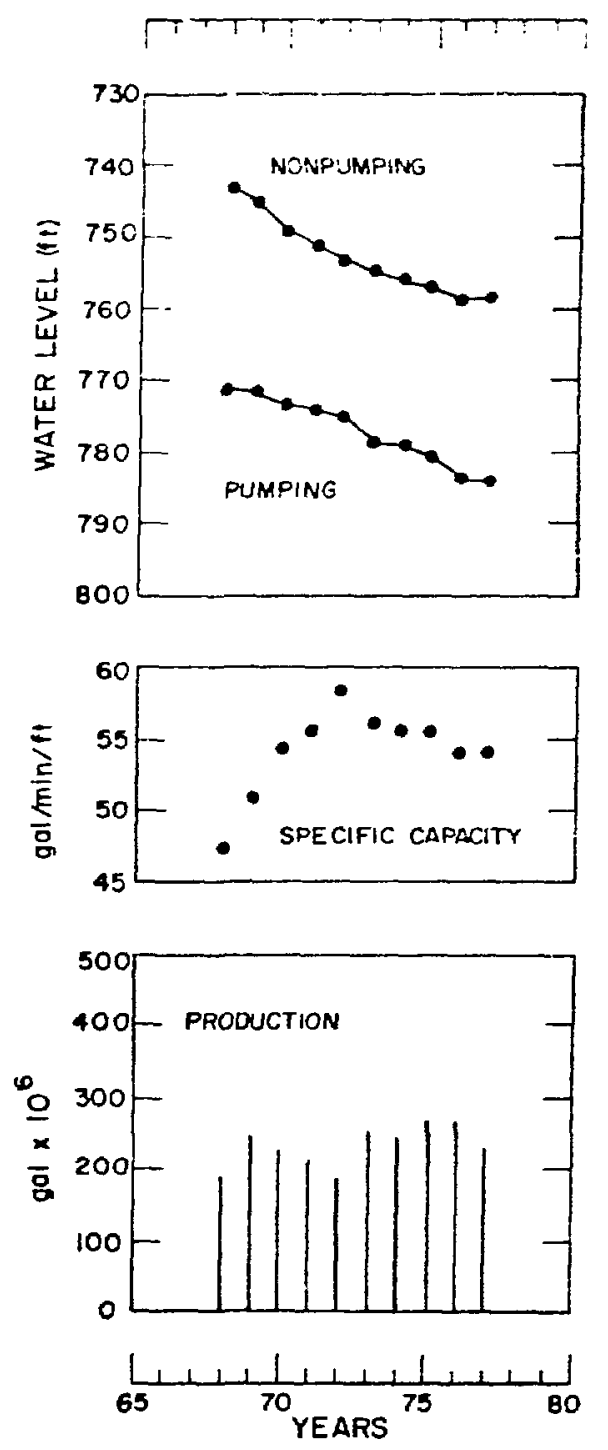

Fig. 19.

Annual average nonpumping and pumping water levels, annual average specific capacity, and annual production, Pajarito Well PM-3. 


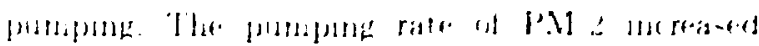

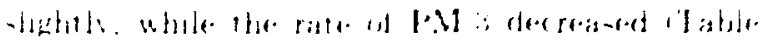

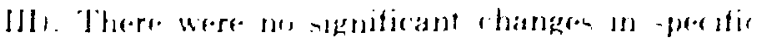
catpacation in the three werlh

\section{WATHE (ANYON (BALESKY}

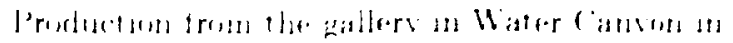

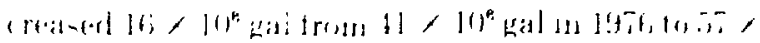

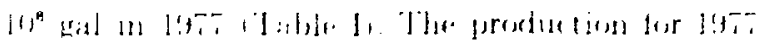

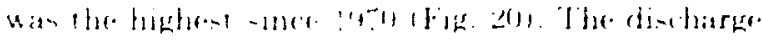

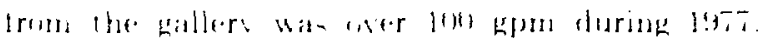

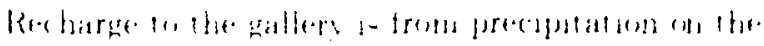

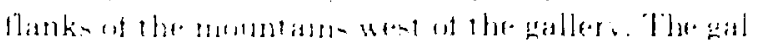

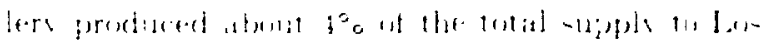

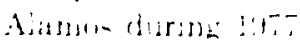

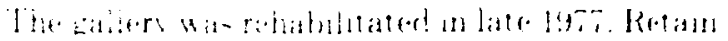

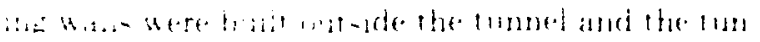
a... - a

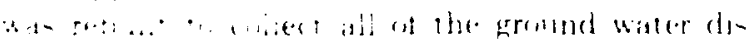
(:: $: 2:-1$

\section{S(MMARY ANI) RECOMMLNI)ATIONS}

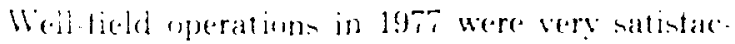
tors. Production trom the three well fieldo rlectined

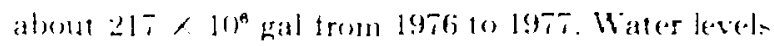
in wells in the los Alamos, Ciuaje, and l'ajarite fields were an expected under current production.
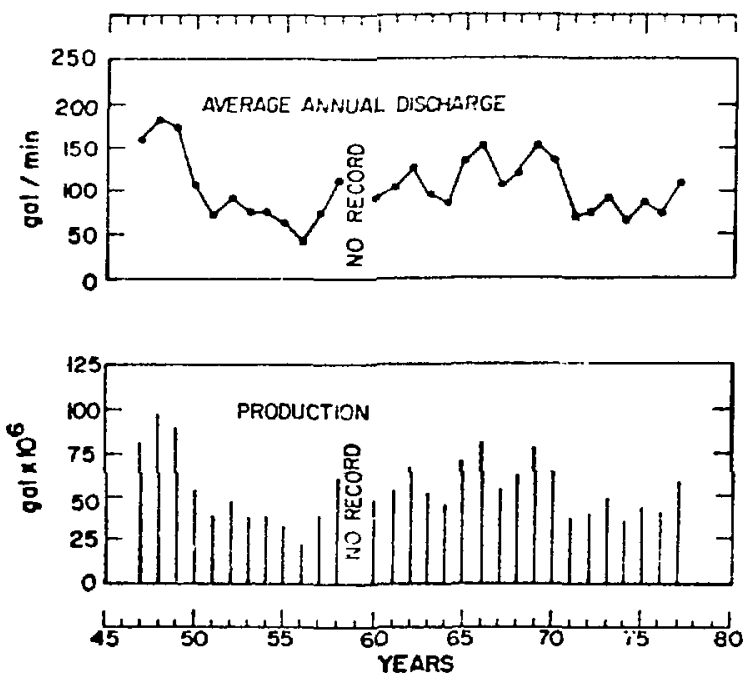

Fig. 20.

Annual average discharge and annual production from the gallery in Water Canyon.

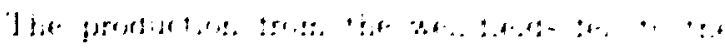

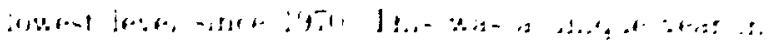

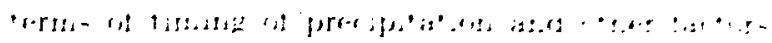

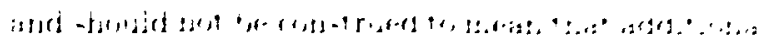

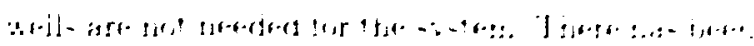

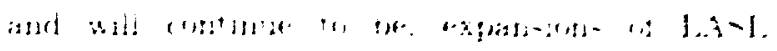

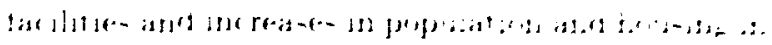

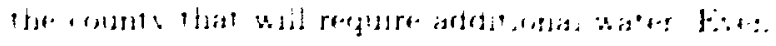

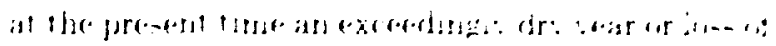

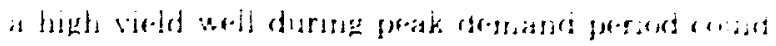

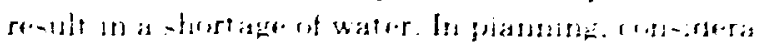

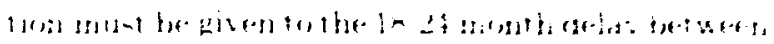

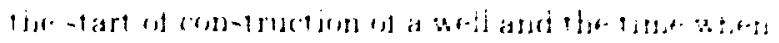

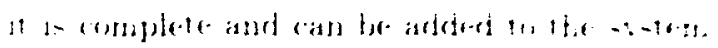

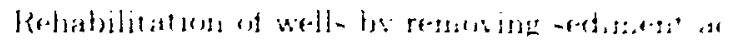

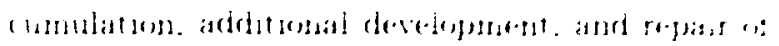

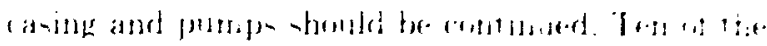

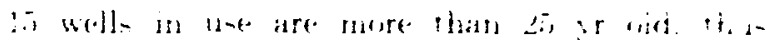

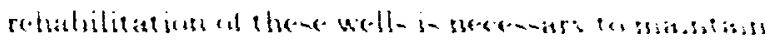

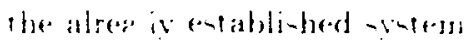

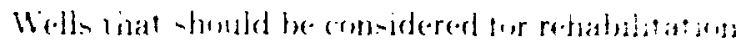

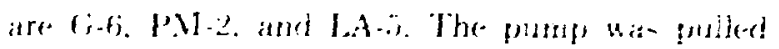

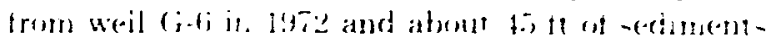
were remered trom the well. The pumplnge sate hat-

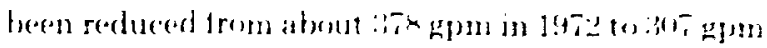

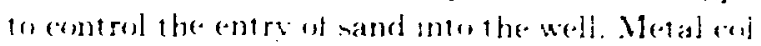
lected in the sand sampler indicate wear woll base shatt or pump. Abent ti $\times 10^{3}$ gal ef water is pumperd (1) Waste at the start of the pumping perade to beep)

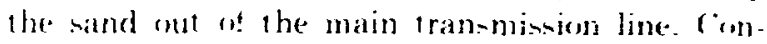
sideration stould be given to installation af a filer ur line to the sand trap at Buserestation l toutilize the water pumped te waste.

Well PM-2 is a high yield well that has been in operation since 1966. The pumping characteristicindicate no problems with the pump: houever. the period of operation (35) $0(0) \mathrm{h}$ ). as recommended lite of pump by the manufacturer. has been exceeded. As the well is a high-yield producer $1 \approx 18^{\circ} \circ$ of tolal productions. the pump should be pulled for inspecijon.

Well $1 . A-5$ was pulled for inspection in $196 \%$. Pumping rates have declined from about $480 \mathrm{gpm}$ to $468 \mathrm{gpm}$ since that period, with only a slight change in specific capacity. Zia $L^{*} / \mathrm{E}$ reports that a larger line shaft is needed to keep the pump in adjustment and that the motor needs to be rewound from 125 to $150 \mathrm{hp}$. as the motor is presently drawing 152 to $155 \mathrm{~A}$ on a $150 \mathrm{~A}$ rating. 


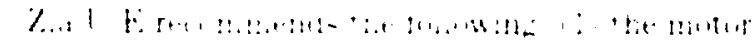

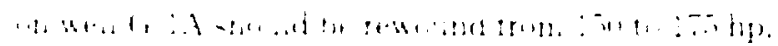

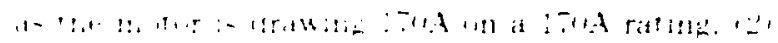

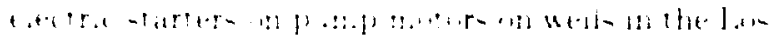

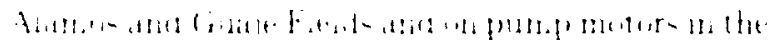

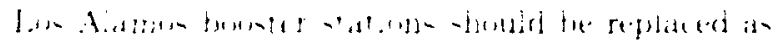

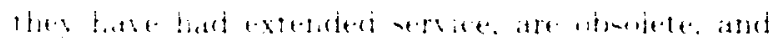

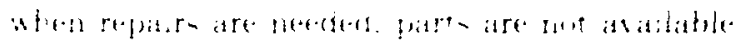

\section{ACKNOWIEI)(BNENTS}

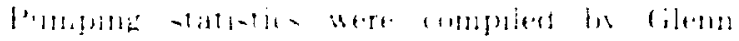

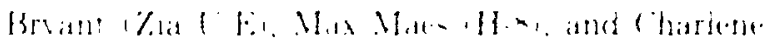

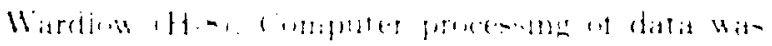

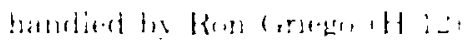

\section{REFERFNCR}

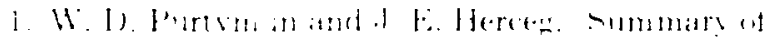

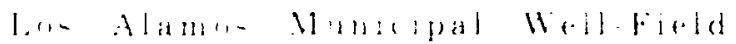

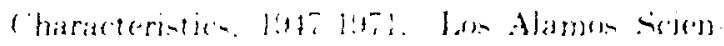

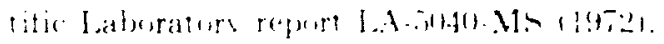

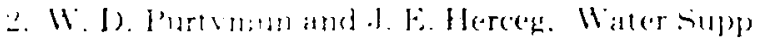

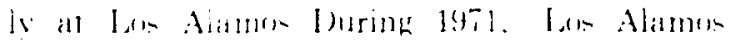

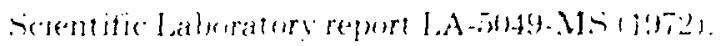

$\therefore$ II. 1). Murtemun and .J. F. Herceg. "Hater siuppIy al lom Aamon Iouring 1972.' Las Alamo

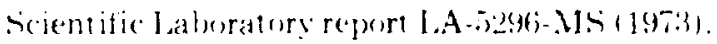

1. 11. 1). Purtymum and .I. H. Herceg. "Mater SuppIy at Ios Alamos During 1973." Ios Alamos

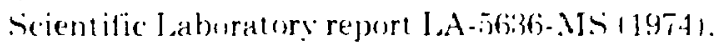

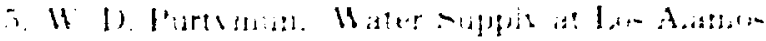

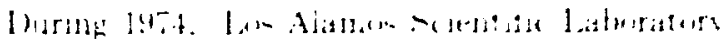

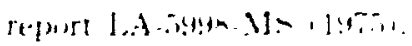

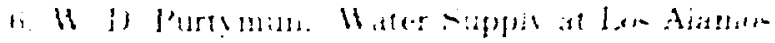

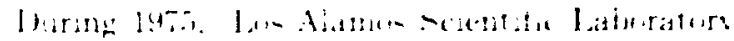

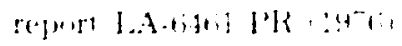

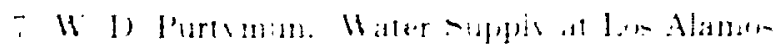

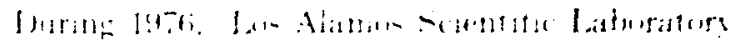

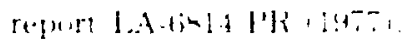

$\therefore$ IR. I. (ushman and 14. I) Turtsmun. An Falualum of the Yield and 18 aler-lexel

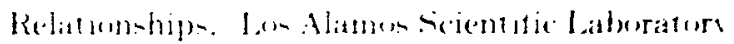

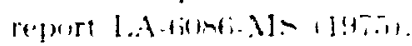

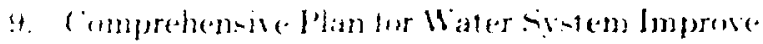
ments. Iar Alamom. Vew Mexice," Gordons Herkenhoft and Amociates. Inc.. Fonginesers and

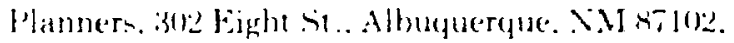
contract Sumber AJi2y-11-2001 11974.

11. 11. 1). Purtymun. "Hydrolugic (haracteristics ot the [os Alamos Wel] Field with Reference to the cocurrence of Arsenic in Well IA-b." Ias Alamos Scientific laboratory report IA-7012Mitigtis. 
APPENIDIX A

MONTHLY AQUIFER CHARACTERISTICS

WELI LA-1

1977

\begin{tabular}{lcc} 
Month & $\begin{array}{c}\text { Water Level } \\
\text { Non Pump } \\
\text { Feet }\end{array}$ \\
\cline { 1 - 1 } Jan & & 50 \\
Feb & & 47 \\
Mar & & 47 \\
Apr & & 54 \\
May & & 76 \\
June & & 105 \\
July & & 110 \\
Aug & & 99 \\
Sep & & 80 \\
Oct & & 85 \\
Nov & & 71 \\
Dec & & 59
\end{tabular}

WELL LA-IB

1977

\begin{tabular}{|c|c|c|c|c|c|c|c|}
\hline \multirow[b]{2}{*}{ Month } & \multirow[b]{2}{*}{$\begin{array}{c}\text { Pump Time } \\
\text { (h) } \\
\end{array}$} & \multirow{2}{*}{$\begin{array}{c}\text { Pumpage } \\
\text { (thousand } \\
\text { gal) }\end{array}$} & \multirow[b]{2}{*}{$\begin{array}{c}\text { Pump Rate } \\
\text { (gpm) }\end{array}$} & \multicolumn{2}{|c|}{ Water Level } & \multirow[b]{2}{*}{$\begin{array}{l}\text { Draw } \\
\text { Down }\end{array}$} & \multirow{2}{*}{$\begin{array}{l}\text { Specific } \\
\text { Capacity } \\
\text { (gpm/ft) }\end{array}$} \\
\hline & & & & $\begin{array}{c}\text { Non Pump } \\
\text { (ft) }\end{array}$ & $\begin{array}{c}\text { Pump } \\
\text { (ft) }\end{array}$ & & \\
\hline Jan & 133 & 4355 & 546 & 22 & 146 & 124 & 4.4 \\
\hline Feb & 129 & 4204 & 543 & 22 & 147 & 125 & 4.3 \\
\hline Mar & 171 & 5612 & 547 & 21 & 145 & 124 & 4.4 \\
\hline Apr & 192 & 6104 & 530 & 24 & 152 & 128 & 4.1 \\
\hline May & 372 & 11322 & 507 & 49 & 171 & 122 & 4.2 \\
\hline June & 434 & 12574 & 483 & 78 & 206 & 128 & 3.8 \\
\hline July & 340 & 9818 & 481 & 85 & 199 & 114 & 4.2 \\
\hline Aug & 298 & 8715 & 487 & 72 & 192 & 120 & 4.1 \\
\hline Sep & 211 & 6281 & 496 & 60 & 180 & 120 & 4.1 \\
\hline Oct & 203 & 6094 & 500 & 56 & 172 & 116 & 4.3 \\
\hline Nov & 157 & 4758 & 505 & 44 & 167 & 123 & 4.1 \\
\hline Dec & 142 & 4364 & 512 & 32 & 123 & 93 & 5.5 \\
\hline
\end{tabular}




\section{APPENDIX A (cont)}

WELI, LA-2

1977

\begin{tabular}{|c|c|c|c|c|c|c|c|}
\hline \multirow[b]{2}{*}{ Month } & \multirow[b]{2}{*}{$\begin{array}{c}\text { Pump Time } \\
\text { (hr) } \\
\end{array}$} & \multirow{2}{*}{$\begin{array}{c}\text { Pumpage } \\
\text { (thousand } \\
\text { (gal) } \\
\end{array}$} & \multirow[b]{2}{*}{$\begin{array}{c}\text { Pump Rate } \\
\text { (gpm) } \\
\end{array}$} & \multicolumn{2}{|c|}{ Water Level } & \multirow[b]{2}{*}{$\begin{array}{l}\text { Draw } \\
\text { Down }\end{array}$} & \multirow{2}{*}{$\begin{array}{l}\text { Specific } \\
\text { Capacity } \\
\text { (gpm/ft) }\end{array}$} \\
\hline & & & & $\begin{array}{l}\begin{array}{c}\text { Non Pump } \\
\text { (ft) }\end{array} \\
\end{array}$ & $\begin{array}{c}\text { Pump } \\
\text { (ft) }\end{array}$ & & \\
\hline Jan & 133 & 2433 & 305 & 90 & 301 & 211 & 1.4 \\
\hline Feb & 128 & 2232 & 291 & 83 & 298 & 215 & 1.4 \\
\hline Mar & 171 & 2998 & 292 & 85 & 301 & 216 & 1.4 \\
\hline Apr & 193 & 3312 & 286 & 94 & 308 & 214 & 1.3 \\
\hline May & 371 & 5595 & 251 & 127 & 320 & 193 & 1.3 \\
\hline June & 435 & 5842 & 224 & 166 & 362 & 196 & 1.1 \\
\hline July & 340 & 4535 & 222 & 166 & 332 & 166 & 1.3 \\
\hline Aug & 298 & 4224 & 236 & 147 & 327 & 180 & 1.3 \\
\hline Sep & 213 & 3204 & 251 & 133 & 318 & 185 & 1.4 \\
\hline Oct & 203 & 3049 & 250 & 127 & 296 & 169 & 1.5 \\
\hline Nov & 157 & 2653 & 282 & 108 & 306 & 198 & 1.4 \\
\hline Dec & 133 & 2433 & 305 & 90 & 301 & 211 & 1.4 \\
\hline
\end{tabular}

WELL, LA-3

1977

\begin{tabular}{|c|c|c|c|c|c|c|c|}
\hline \multirow[b]{2}{*}{ Month } & \multirow[b]{2}{*}{$\begin{array}{c}\text { Pump Time } \\
\text { (h) }\end{array}$} & \multirow{2}{*}{$\begin{array}{c}\text { Pumpage } \\
\text { (thousand } \\
\text { gal) } \\
\end{array}$} & \multirow[b]{2}{*}{$\begin{array}{c}\text { Pump Rate } \\
\text { (gpm) }\end{array}$} & \multicolumn{2}{|c|}{ Water Level } & \multirow[b]{2}{*}{$\begin{array}{l}\text { Draw } \\
\text { Down } \\
\end{array}$} & \multirow{2}{*}{$\begin{array}{l}\text { Specific } \\
\text { Capacity } \\
\text { (gpm/ft } \\
\end{array}$} \\
\hline & & & & $\begin{array}{c}\text { Non Pump } \\
\text { (ft) }\end{array}$ & $\begin{array}{c}\text { Pump } \\
\text { (ft) }\end{array}$ & & \\
\hline Jan & 133 & 2644 & 331 & 66 & 250 & 184 & 1.8 \\
\hline Feb & 128 & 2526 & 329 & 61 & 246 & 185 & 1.8 \\
\hline Mar & 171 & 3382 & 330 & 63 & 247 & 184 & 1.8 \\
\hline Apr & 192 & 3687 & 320 & 69 & 248 & 179 & 1.8 \\
\hline May & 370 & 6299 & 284 & 95 & 253 & 158 & 1.8 \\
\hline June & 435 & 6101 & 234 & 122 & 258 & 136 & 1.7 \\
\hline July & 340 & 5118 & 251 & 125 & 258 & 133 & 1.9 \\
\hline Aug & 298 & 4821 & 270 & 113 & 256 & 143 & 1.9 \\
\hline Sep & 211 & 3665 & 289 & 102 & 251 & 149 & 1.9 \\
\hline Oct & 202 & 3564 & 294 & 95 & 254 & 159 & 1.8 \\
\hline Nov & 157 & 2875 & 305 & 82 & 253 & 171 & 1.8 \\
\hline Dec & 142 & 2644 & 310 & 72 & 205 & 133 & 2.3 \\
\hline
\end{tabular}


WELI LA-4

1977

\begin{tabular}{|c|c|c|c|c|c|c|c|}
\hline \multirow[b]{2}{*}{ Month } & \multirow[b]{2}{*}{$\begin{array}{c}\text { Pump Time } \\
\text { (hr) }\end{array}$} & \multirow{2}{*}{$\begin{array}{c}\text { Pumpage } \\
\text { (thousand } \\
\text { gal) } \\
\end{array}$} & \multirow[b]{2}{*}{$\begin{array}{c}\text { Pump Rate } \\
\text { (gpm) }\end{array}$} & \multicolumn{2}{|c|}{ Water Level } & \multirow[b]{2}{*}{$\begin{array}{l}\text { Draw } \\
\text { Down }\end{array}$} & \multirow{2}{*}{$\begin{array}{l}\text { Specific } \\
\text { Capacity } \\
\text { (gpm/ft) }\end{array}$} \\
\hline & & & & $\begin{array}{c}\text { Non Pump } \\
\text { (ft) }\end{array}$ & $\begin{array}{c}\text { Pump } \\
\text { (ft) }\end{array}$ & & \\
\hline Jan & 132 & 4746 & 599 & 264 & 361 & 97 & 6.2 \\
\hline Feb & 123 & 4437 & 601 & 263 & 360 & 97 & 6.2 \\
\hline Mar & 162 & 5844 & 601 & 261 & 360 & 99 & 6.1 \\
\hline Apr & 182 & 6558 & 601 & 266 & 365 & 99 & 6.1 \\
\hline May & 362 & 12778 & 588 & 278 & 376 & 98 & 6.0 \\
\hline June & 446 & 15105 & 564 & 304 & 395 & 91 & 6.2 \\
\hline July & 342 & 11787 & 574 & 302 & 395 & 9.3 & 6.2 \\
\hline Aug & 280 & 9763 & 581 & 295 & 387 & 92 & 6.3 \\
\hline Sep & 211 & 7440 & 588 & 281 & 375 & 94 & 6.3 \\
\hline Oct & 199 & 7061 & 591 & 281 & 375 & 94 & 6.3 \\
\hline Nov & 164 & 5866 & 596 & 274 & 369 & 95 & 6.3 \\
\hline Dec & 138 & 4976 & 601 & 270 & 366 & 96 & 6.3 \\
\hline
\end{tabular}

\footnotetext{
WELL LA-5

1977
}

\begin{tabular}{|c|c|c|c|c|c|c|c|}
\hline \multirow[b]{2}{*}{ Month } & \multirow[b]{2}{*}{$\begin{array}{c}\text { Pump Time } \\
\text { (h) } \\
\end{array}$} & \multirow{2}{*}{$\begin{array}{c}\text { Pumpage } \\
\text { (thousand } \\
\text { gal) } \\
\end{array}$} & \multirow[b]{2}{*}{$\begin{array}{c}\text { Pump Rate } \\
\text { (gpm) } \\
\end{array}$} & \multicolumn{2}{|c|}{ Water Level } & \multirow[b]{2}{*}{$\begin{array}{l}\text { Draw } \\
\text { Down }\end{array}$} & \multirow{2}{*}{$\begin{array}{l}\text { Specific } \\
\text { Capacity } \\
(\mathrm{gpm} / \mathrm{ft})\end{array}$} \\
\hline & & & & $\begin{array}{c}\text { Non Pump } \\
\text { (ft) }\end{array}$ & $\begin{array}{c}\text { Pump } \\
\text { (ft) }\end{array}$ & & \\
\hline Jan & 132 & 3803 & 480 & 143 & 302 & 159 & 3.0 \\
\hline Feb & 123 & 3537 & 479 & 141 & 302 & 161 & 3.0 \\
\hline Mar & 164 & 4714 & 479 & 139 & 300 & 161 & 3.0 \\
\hline Apr & 173 & 4973 & 479 & 141 & 301 & 160 & 3.0 \\
\hline May & 285 & 7953 & 465 & 144 & 306 & 162 & 2.9 \\
\hline June & 442 & 11939 & 450 & 157 & 310 & 153 & 2.9 \\
\hline July & 342 & 9408 & 458 & 157 & 311 & 154 & 3.0 \\
\hline Aug & 292 & 8125 & 464 & 152 & 304 & 152 & 3.1 \\
\hline Sep & 213 & 6001 & 470 & 149 & 302 & 153 & 3.1 \\
\hline Oct & 199 & 5667 & 475 & 149 & 303 & 154 & 3.1 \\
\hline Nov & 163 & 4670 & 478 & 145 & 301 & 156 & 3.1 \\
\hline Dec & 137 & 3979 & 484 & 145 & 299 & 154 & 3.1 \\
\hline
\end{tabular}




\section{APPENDIX A (cont)}

WELL LA-f

1977

\begin{tabular}{lcc} 
Month & $\begin{array}{c}\text { Won Pump } \\
\text { (ft) }\end{array}$ \\
\cline { 1 - 1 } Jan & & 81 \\
Feb & & $\ldots$ \\
Mar & & 75 \\
Apr & & 76 \\
May & & 75 \\
June & & 79 \\
July & & 87 \\
Aug & & 87 \\
Sep & & 87 \\
Oct & & 86 \\
Nov & & 82 \\
Dec & & 82
\end{tabular}

WELL G-1

1977

\begin{tabular}{|c|c|c|c|c|c|c|c|}
\hline \multirow[b]{2}{*}{ Month } & \multirow[b]{2}{*}{$\begin{array}{c}\text { Pump Time } \\
\text { (h) } \\
\end{array}$} & \multirow{2}{*}{$\begin{array}{c}\text { Pumpage } \\
\text { (thousand } \\
\text { gal) } \\
\end{array}$} & \multirow[b]{2}{*}{$\begin{array}{c}\text { Pump Rate } \\
\text { (gpm) }\end{array}$} & \multicolumn{2}{|c|}{ Water Level } & \multirow[b]{2}{*}{$\begin{array}{l}\text { Draw } \\
\text { Down }\end{array}$} & \multirow{2}{*}{$\begin{array}{l}\text { Specific } \\
\text { Capacity } \\
\text { (gpm/ft) }\end{array}$} \\
\hline & & & & $\begin{array}{c}\text { Non Pump } \\
\text { (ft) }\end{array}$ & $\begin{array}{c}\text { Pump } \\
\text { (ft) }\end{array}$ & & \\
\hline Jan & 140 & 3172 & 378 & $\ldots$ & $\cdots$ & $\cdots$ & $\ldots$ \\
\hline Feb & 101 & 2250 & 371 & -.. & $\ldots$ & $-\cdot$ & -- \\
\hline Mar & 0 & 0 & 0 & ..- & $\cdots$ & $\cdots$ & -- \\
\hline Apr & 175 & 3930 & 374 & 272 & 419 & 147 & 2.5 \\
\hline May & 402 & 8742 & 362 & 274 & 419 & 145 & 2.5 \\
\hline June & 473 & 9581 & 338 & 280 & 429 & 149 & 2.3 \\
\hline July & 355 & 7267 & 341 & 280 & 433 & 153 & 2.2 \\
\hline Aug & 293 & 6045 & 344 & 279 & 432 & 153 & 2.2 \\
\hline Sep & 215 & 4530 & 351 & 274 & 426 & 152 & 2.3 \\
\hline Oct & 236 & 4994 & 353 & 277 & 429 & 152 & 2.3 \\
\hline Nov & 178 & 3803 & 356 & 272 & 423 & 151 & 2.4 \\
\hline Dec & 166 & 3558 & 357 & 271 & 420 & 149 & 2.4 \\
\hline
\end{tabular}


WELL G-1A

1977

\begin{tabular}{|c|c|c|c|c|c|c|c|}
\hline \multirow[b]{2}{*}{ Month } & \multirow[b]{2}{*}{$\begin{array}{c}\text { Pump Time } \\
\text { (h) }\end{array}$} & \multirow{2}{*}{$\begin{array}{c}\text { Pumpage } \\
\text { (thousand } \\
\text { gal) }\end{array}$} & \multirow[b]{2}{*}{$\begin{array}{c}\text { Pump Fate } \\
\text { (gpm) }\end{array}$} & \multicolumn{2}{|c|}{ Water Level } & \multirow[b]{2}{*}{$\begin{array}{l}\text { Draw } \\
\text { Down }\end{array}$} & \multirow{2}{*}{$\begin{array}{l}\text { Specific } \\
\text { Capacity } \\
\text { (gpm/ft) }\end{array}$} \\
\hline & & & & $\begin{array}{l}\text { Non Pump } \\
\text { (ft) }\end{array}$ & $\begin{array}{c}\text { Pump } \\
\text { (ft) }\end{array}$ & & \\
\hline Jan & 133 & 4040 & 506 & 298 & 348 & 50 & 10.1 \\
\hline Feb & 152 & 4491 & 492 & 299 & 348 & 49 & 10.0 \\
\hline Mar & 166 & 4977 & 500 & 296 & 341 & 45 & 11.1 \\
\hline Apr & 174 & 5372 & 515 & 297 & 346 & 49 & 10.5 \\
\hline May & 402 & 12153 & 504 & 300 & 350 & 50 & 10.1 \\
\hline June & 473 & 14248 & 502 & 308 & 356 & 48 & 10.5 \\
\hline July & 355 & 10599 & 498 & 309 & 358 & 49 & 10.2 \\
\hline Aug & 293 & 8785 & 500 & 306 & 355 & 49 & 10.2 \\
\hline Sep & 215 & 6428 & 498 & 303 & 354 & 51 & 9.8 \\
\hline Oct & 236 & 7177 & 507 & 303 & 350 & 47 & 10.8 \\
\hline Nov & 178 & 5428 & 508 & 302 & 347 & 45 & 11.3 \\
\hline Dec & 165 & 4999 & 505 & 300 & 347 & 47 & 10.7 \\
\hline
\end{tabular}

WELL G-2

1977

\begin{tabular}{|c|c|c|c|c|c|c|c|}
\hline \multirow[b]{2}{*}{ Month } & \multirow[b]{2}{*}{$\begin{array}{c}\text { Pump Time } \\
\text { (h) }\end{array}$} & \multirow{2}{*}{$\begin{array}{c}\text { Pumpage } \\
\text { (thousand } \\
\text { gal) } \\
\end{array}$} & \multirow[b]{2}{*}{$\begin{array}{c}\text { Pump Rate } \\
\text { (gpm) }\end{array}$} & \multicolumn{2}{|c|}{ Water Level } & \multirow[b]{2}{*}{$\begin{array}{l}\text { Draw } \\
\text { Down } \\
\end{array}$} & \multirow{2}{*}{$\begin{array}{l}\text { Specific } \\
\text { Capacity } \\
(\mathrm{gpm} / \mathrm{ft}) \\
\end{array}$} \\
\hline & & & & $\begin{array}{c}\text { Non Pump } \\
\text { (ft) } \\
\end{array}$ & $\underset{\text { (ft) }}{\text { Pump }}$ & & \\
\hline Jan & 136 & 3785 & 464 & 341 & 387 & 46 & 10.1 \\
\hline Feb & 176 & 4840 & 458 & 341 & 386 & 45 & 10.2 \\
\hline Mar & 178 & 4901 & 459 & 341 & 383 & 42 & 10.9 \\
\hline Apr & 174 & 4901 & 469 & 341 & 385 & 44 & 10.7 \\
\hline May & 402 & 10731 & 445 & 344 & 387 & 43 & 10.3 \\
\hline June & 473 & 12317 & 434 & 354 & 396 & 42 & 10.3 \\
\hline July & 355 & 9396 & 441 & 354 & 394 & 40 & 11.0 \\
\hline Aug & 293 & 7816 & 445 & 352 & 392 & 40 & 11.1 \\
\hline Sep & 215 & 5809 & 450 & 349 & 390 & 41 & 11.0 \\
\hline Oct & 236 & 6452 & 456 & 349 & 386 & 37 & 12.3 \\
\hline Nov & 177 & 4915 & 463 & 346 & 388 & 42 & 11.0 \\
\hline Dec & 166 & 4562 & 458 & 345 & 387 & 42 & 10.9 \\
\hline
\end{tabular}


WELL, (;-3

1977

\begin{tabular}{|c|c|c|c|c|c|c|c|}
\hline \multirow[b]{2}{*}{ Month } & \multirow[b]{2}{*}{$\begin{array}{c}\text { Pump Time } \\
\text { (h) }\end{array}$} & \multirow{2}{*}{$\begin{array}{c}\text { Pumpage } \\
\text { (thousand } \\
\text { gal) } \\
\end{array}$} & \multirow[b]{2}{*}{$\begin{array}{c}\text { Pump Rate } \\
\text { (gpm) }\end{array}$} & \multicolumn{2}{|c|}{ Water Level } & \multirow[b]{2}{*}{$\begin{array}{l}\text { Draw } \\
\text { Down }\end{array}$} & \multirow{2}{*}{$\begin{array}{l}\text { Specific } \\
\text { Capacity } \\
\text { (gpm/ft) }\end{array}$} \\
\hline & & & & $\begin{array}{l}\text { Non Pump } \\
\text { (ft) }\end{array}$ & $\begin{array}{c}\text { Pump } \\
\text { (ft) }\end{array}$ & & \\
\hline Jan & 142 & 4026 & $4: 3$ & 373 & $46 \%$ & 94 & 5.0 \\
\hline Feb & 161 & 4624 & 479 & 352 & 442 & 90 & 5.3 \\
\hline Mar & 166 & 4660 & 468 & 350 & 444 & 94 & 5.0 \\
\hline Apr & 175 & 4979 & 474 & 351 & 447 & 96 & 4.9 \\
\hline May & 396 & 10746 & 452 & 364 & 456 & 92 & 4.9 \\
\hline June & 479 & 12281 & 427 & 387 & 473 & 86 & 5.0 \\
\hline July & 357 & 9140 & 427 & 389 & 477 & 88 & 4.8 \\
\hline Aug & 291 & 7529 & $4: 31$ & 381 & 471 & 90 & 4.8 \\
\hline Sep & 215 & 5651 & $4: 38$ & 372 & 467 & 95 & 4.6 \\
\hline Oct & $2: 34$ & 6138 & 437 & 371 & 466 & 95 & 4.6 \\
\hline Nov & 178 & 4708 & 441 & 364 & 462 & 98 & 4.5 \\
\hline Dec & 167 & 4440 & 443 & 359 & 485 & 126 & 3.5 \\
\hline
\end{tabular}

WELL G-4

1977

\begin{tabular}{|c|c|c|c|c|c|c|c|}
\hline \multirow[b]{2}{*}{ Month } & \multirow[b]{2}{*}{$\begin{array}{c}\text { Pump Time } \\
\text { (h) }\end{array}$} & \multirow{2}{*}{$\begin{array}{c}\text { Pumpage } \\
\text { (thousand } \\
\text { gal) } \\
\end{array}$} & \multirow[b]{2}{*}{$\begin{array}{c}\text { Pump Rate } \\
\text { (gpm) }\end{array}$} & \multicolumn{2}{|c|}{ Water Level } & \multirow[b]{2}{*}{$\begin{array}{l}\text { Draw } \\
\text { Down }\end{array}$} & \multirow{2}{*}{$\begin{array}{l}\text { Specific } \\
\text { Capacity } \\
\text { (gpm/fit) }\end{array}$} \\
\hline & & & & $\begin{array}{c}\text { Non Pump } \\
\text { (ft) }\end{array}$ & $\begin{array}{c}\text { Pump } \\
\text { (ft) }\end{array}$ & & \\
\hline Jan & 127 & 2802 & 368 & 396 & 582 & 186 & 2.0 \\
\hline Feb & 171 & 3828 & 373 & 395 & 583 & 188 & 2.0 \\
\hline Mar & 176 & 4030 & 382 & 397 & 579 & 182 & 2.1 \\
\hline Apr & 175 & 3850 & 367 & 397 & 582 & 185 & 2.0 \\
\hline May & 396 & 8439 & 355 & 405 & 594 & 189 & 1.9 \\
\hline June & 470 & 9232 & 327 & 424 & 595 & 171 & 1.9 \\
\hline July & 357 & 7336 & 342 & 425 & 595 & 170 & 2.0 \\
\hline Aug & 290 & 6044 & 347 & 417 & 595 & 178 & 2.0 \\
\hline Sep & 215 & 4551 & 353 & 406 & 590 & 184 & 1.9 \\
\hline Oct & 234 & 4939 & 352 & 406 & 588 & 182 & 1.9 \\
\hline Nov & 178 & 3846 & 360 & 405 & 590 & 185 & 1.9 \\
\hline Dec & 165 & 3466 & 350 & 401 & 591 & 190 & 1.8 \\
\hline
\end{tabular}




\section{APPENDIX A (cont)}

WIELL, ( $i-5)$

1977

\begin{tabular}{|c|c|c|c|c|c|c|c|}
\hline \multirow[b]{2}{*}{ Month } & \multirow[b]{2}{*}{$\begin{array}{c}\text { Pump Tine } \\
\text { (h) }\end{array}$} & \multirow{2}{*}{$\begin{array}{c}\text { Pumpage } \\
\text { (thousand } \\
\text { gal) } \\
\end{array}$} & \multirow[b]{2}{*}{$\begin{array}{c}\text { Pump Rate } \\
\text { (gpm) }\end{array}$} & \multicolumn{2}{|c|}{ Water Level } & \multirow[b]{2}{*}{$\begin{array}{l}\text { Draw } \\
\text { Down }\end{array}$} & \multirow{2}{*}{$\begin{array}{c}\text { Specific } \\
\text { Capacity } \\
\text { (gpm/ft) }\end{array}$} \\
\hline & & & & $\begin{array}{c}\text { Non Pump } \\
\text { (ft) }\end{array}$ & $\begin{array}{c}\text { Pump } \\
\text { (ft) } \\
\end{array}$ & & \\
\hline Jan & 138 & 4526 & 547 & $4: 37$ & 496 & 59 & 9.3 \\
\hline Feb & 64 & 2077 & 541 & 4.38 & 496 & 58 & 9.3 \\
\hline Mar & 176 & 5761 & 546 & 4.37 & 497 & 60 & 9.1 \\
\hline Apr & 175 & 57.38 & 546 & 4.37 & 497 & 60 & 9.1 \\
\hline May & 393 & 12598 & 5.34 & 444 & 50.5 & 61 & 8.8 \\
\hline June & 477 & 14995 & 524 & 457 & 522 & 65 & 8.1 \\
\hline July & 357 & $11: 343$ & 530 & 4.56 & 516 & 60 & 8.8 \\
\hline Aug & 290 & 9270 & 533 & 450 & 512 & 62 & 8.6 \\
\hline Sep & 215 & 6951 & 5.39 & 446 & 506 & 60 & 9.0 \\
\hline Oct & 234 & 7597 & 541 & 446 & 506 & 60 & 9.0 \\
\hline Nov & 178 & 5819 & 545 & 442 & 501 & 59 & 9.2 \\
\hline Dec & 166 & 53.397 & 542 & 439 & 499 & 60 & 9.0 \\
\hline
\end{tabular}

WELL G-6

1977

\begin{tabular}{|c|c|c|c|c|c|c|c|}
\hline \multirow[b]{2}{*}{ Month } & \multirow[b]{2}{*}{$\begin{array}{c}\text { Pump Time } \\
\text { (h) }\end{array}$} & \multirow{2}{*}{$\begin{array}{c}\text { Pumpage } \\
\text { (thousand } \\
\text { gal) } \\
\end{array}$} & \multirow[b]{2}{*}{$\begin{array}{c}\text { Pump Rate } \\
\text { (gpm) } \\
\end{array}$} & \multicolumn{2}{|c|}{ Water Level } & \multirow[b]{2}{*}{$\begin{array}{l}\text { Draw } \\
\text { Down }\end{array}$} & \multirow{2}{*}{$\begin{array}{l}\text { Specific } \\
\text { Capacity } \\
\text { (gpm/ft) }\end{array}$} \\
\hline & & & & $\begin{array}{c}\text { Non Pump } \\
\text { (ft) } \\
\end{array}$ & $\begin{array}{c}\text { Pump } \\
\text { (ft) }\end{array}$ & & \\
\hline Jan & 142 & 2729 & 320 & 579 & 661 & 82 & 3.9 \\
\hline Feb & 160 & 3181 & 331 & 578 & 653 & 75 & 4.4 \\
\hline Mar & 175 & 3436 & 327 & 579 & 650 & 71 & 4.6 \\
\hline Apr & 172 & 3420 & 331 & 579 & 655 & 76 & 4.4 \\
\hline May & 394 & 7366 & 312 & 585 & 660 & 75 & 4.2 \\
\hline June & 477 & 8550 & 299 & 604 & 674 & 70 & 4.3 \\
\hline July & 353 & 6344 & 300 & 603 & 678 & 75 & 4.0 \\
\hline Aug & 291 & 5265 & 302 & 594 & 666 & 72 & 4.2 \\
\hline Sep & 212 & 3810 & 300 & 586 & 658 & 72 & 4.2 \\
\hline Oct & 234 & 4171 & 297 & 588 & 656 & 68 & 4.4 \\
\hline Nov & 178 & 3225 & 302 & 582 & 650 & 68 & $\$ .4$ \\
\hline Dec & 166 & 2949 & 296 & 580 & 648 & 68 & 4.4 \\
\hline
\end{tabular}




\title{
APPENDIX A (cont)
}

\author{
WELL PM-I \\ 1977
}

\begin{tabular}{|c|c|c|c|c|c|c|c|}
\hline \multirow[b]{2}{*}{ Month } & \multirow[b]{2}{*}{$\begin{array}{c}\text { Pump Time } \\
\text { (h) }\end{array}$} & \multirow{2}{*}{$\begin{array}{c}\text { Pumpage } \\
\text { (thousand } \\
\text { gal) } \\
\end{array}$} & \multirow[b]{2}{*}{$\begin{array}{c}\text { Pump Rate } \\
\text { (gpm) }\end{array}$} & \multicolumn{2}{|c|}{ Water Level } & \multirow[b]{2}{*}{$\begin{array}{l}\text { Draw } \\
\text { Down }\end{array}$} & \multirow{2}{*}{$\begin{array}{r}\text { Specific } \\
\text { Capacity } \\
\text { (gpm-ft) } \\
\end{array}$} \\
\hline & & & & $\begin{array}{c}\begin{array}{c}\text { Non Pump } \\
\text { (ft) }\end{array} \\
\end{array}$ & $\begin{array}{c}\text { Pump } \\
\text { (ft) } \\
\end{array}$ & & \\
\hline Jan & 195 & 7000.3 & 599 & 742 & 762 & 20 & 29.9 \\
\hline Feb & 161 & 5807 & 601 & 74.3 & 764 & 21 & 28.6 \\
\hline Mar & 153 & 5434 & 592 & 742 & 762 & 20 & 29.6 \\
\hline Apr & 179 & $6: 373$ & 593 & 742 & 764 & 22 & 27.0 \\
\hline May & 430 & 15519 & 602 & 744 & 769 & 25 & 24.1 \\
\hline June & 440 & 15842 & 600 & 751 & 773 & 22 & 27.3 \\
\hline July & 339 & 12088 & 594 & 748 & 772 & 24 & 24.8 \\
\hline Aug & $3: 31$ & 11060 & 557 & 747 & 771 & 24 & 23.2 \\
\hline Sep & 268 & $95 \overline{5} 6$ & 594 & 747 & 770 & 23 & 25.8 \\
\hline Oct & 219 & 7765 & 591 & 745 & 768 & 23 & 25.7 \\
\hline $\operatorname{Pov}$ & 125 & 4367 & 582 & 744 & 766 & 22 & 26.5 \\
\hline Dec & 129 & 4571 & 591 & 742 & 763 & 21 & 28.1 \\
\hline
\end{tabular}

WELL PM-2

1977

\begin{tabular}{|c|c|c|c|c|c|c|c|}
\hline \multirow[b]{2}{*}{ Month } & \multirow[b]{2}{*}{$\begin{array}{c}\text { Pump Time } \\
\text { (h) }\end{array}$} & \multirow{2}{*}{$\begin{array}{c}\text { Pumpage } \\
\text { (thousand } \\
\text { gal) } \\
\end{array}$} & \multirow[b]{2}{*}{$\begin{array}{c}\text { Pump Rate } \\
\text { (gpm) }\end{array}$} & \multicolumn{2}{|c|}{ Water Level } & \multirow[b]{2}{*}{$\begin{array}{l}\text { Draw } \\
\text { Down } \\
\end{array}$} & \multirow{2}{*}{$\begin{array}{l}\text { Specific } \\
\text { Capacity } \\
\text { (gpm/ft) }\end{array}$} \\
\hline & & & & $\begin{array}{l}\text { Non Pump } \\
\text { (ft) }\end{array}$ & $\begin{array}{c}\text { Pump } \\
\text { (ft) }\end{array}$ & & \\
\hline $\operatorname{Jan}$ & 257 & 21178 & 1373 & 868 & 922 & 54 & 25.4 \\
\hline Feb & 309 & 25502 & 1376 & 869 & 924 & 55 & 25.0 \\
\hline Mar & 394 & 32511 & 1375 & 872 & 928 & 56 & 24.6 \\
\hline Apr & 299 & 24666 & 1375 & 870 & 928 & 58 & 23.7 \\
\hline May & 242 & 19988 & 1377 & 868 & 927 & 59 & 23.3 \\
\hline June & 320 & 26366 & 1373 & 870 & 930 & 60 & 22.9 \\
\hline July & 256 & 21089 & 1373 & 869 & 926 & 57 & 24.1 \\
\hline Aug & 292 & 24046 & 1372 & 868 & 925 & 57 & 24.1 \\
\hline Sep & 204 & 16868 & 1378 & 865 & 918 & 53 & 26.0 \\
\hline Oct & 232 & 19175 & 1378 & 865 & 917 & 52 & 26.5 \\
\hline Nov & 205 & 16938 & 1377 & 866 & 920 & 54 & 25.5 \\
\hline Dec & 296 & 24436 & 1376 & 867 & 925 & 58 & 23.7 \\
\hline
\end{tabular}


WELL PM-3

1977

\begin{tabular}{|c|c|c|c|c|c|c|c|}
\hline \multirow[b]{2}{*}{ Month } & \multirow[b]{2}{*}{$\begin{array}{c}\text { Pump Time } \\
\text { (h) }\end{array}$} & \multirow{2}{*}{$\begin{array}{c}\text { Pumpage } \\
\text { (thousand } \\
\text { gal) } \\
\end{array}$} & \multirow[b]{2}{*}{$\begin{array}{c}\text { Pump Rate } \\
\text { (gpm) } \\
\end{array}$} & \multicolumn{2}{|c|}{ Water Level } & \multirow[b]{2}{*}{$\begin{array}{l}\text { Draw } \\
\text { Down } \\
\end{array}$} & \multirow{2}{*}{$\begin{array}{l}\text { Specific } \\
\text { Capacity } \\
\text { (gpm/ft) }\end{array}$} \\
\hline & & & & $\begin{array}{c}\text { Non Pump } \\
\text { (ft) }\end{array}$ & $\begin{array}{c}\text { Pump } \\
\text { (ft) }\end{array}$ & & \\
\hline Jan & 98 & 8149 & 1386 & 757 & 780 & 23 & 60.3 \\
\hline Feb & 10.3 & 8626 & 1396 & 757 & 783 & 26 & 53.7 \\
\hline Mar & 155 & 12941 & 1392 & 757 & 783 & 26 & 53.5 \\
\hline Apr & 179 & 15083 & 1404 & 757 & 784 & 27 & 52.0 \\
\hline May & 434 & 36808 & 1414 & 757 & 785 & 28 & 50.5 \\
\hline June & 444 & 37665 & 1414 & 760 & 786 & 26 & 54.4 \\
\hline July & 337 & 28527 & 1411 & 760 & 785 & 25 & 56.4 \\
\hline Aug & 311 & 26258 & 1407 & 759 & $78 \hat{0}$ & 27 & 52.1 \\
\hline Sep & 268 & 22645 & 1408 & 760 & 785 & 25 & 56.3 \\
\hline Oct & 220 & 18488 & 1401 & 759 & 785 & 26 & 53.9 \\
\hline Nov & 125 & 10476 & 1397 & 757 & 783 & 26 & 53.7 \\
\hline Dec & 118 & 9860 & 1393 & 757 & 781 & 24 & 58.0 \\
\hline
\end{tabular}

WATER CANYON GALLERY

1977

\begin{tabular}{|c|c|c|c|}
\hline Month & $\begin{array}{c}\text { Pump Time } \\
\text { (h) }\end{array}$ & $\begin{array}{c}\text { Pumpage } \\
\text { (thousand } \\
\text { gal) } \\
\end{array}$ & $\begin{array}{c}\text { Rate } \\
\text { (gpm) }\end{array}$ \\
\hline Jan & 744 & 4631 & 104 \\
\hline Feb & 672 & 4497 & 112 \\
\hline Mar & 744 & 2936 & 66 \\
\hline Apr & 720 & 4344 & 101 \\
\hline May & 744 & 5049 & 113 \\
\hline June & 720 & 5942 & 138 \\
\hline July & 744 & 3315 & 74 \\
\hline Aug & 744 & 2662 & 60 \\
\hline Sep & 720 & 7965 & 184 \\
\hline Oct & 744 & 7065 & 158 \\
\hline Nov & 720 & 5683 & 132 \\
\hline Dec & 744 & 3221 & 72 \\
\hline
\end{tabular}


APPENDIX B

ANNUAL AQUIFER CHARACTERISTICS

WELIL LA-1

\begin{tabular}{|c|c|c|c|c|}
\hline Year & $\begin{array}{c}\text { Pump Time } \\
\text { (h) }\end{array}$ & $\begin{array}{c}\text { Pumpage } \\
\text { (million } \\
\text { (gal) }\end{array}$ & $\begin{array}{c}\text { Pump Rate } \\
\text { (gpm) }\end{array}$ & $\begin{array}{c}\text { Water Level } \\
\text { Non Pump } \\
\text { (ft) } \\
\end{array}$ \\
\hline 1947 & 3468 & 54.0 & 259.5 & $\ldots$ \\
\hline 1948 & 2988 & 34.7 & 193.6 & $\ldots$ \\
\hline 1949 & 1361 & 26.7 & 327.0 & $\ldots$ \\
\hline 1950 & 563 & 10.5 & 310.8 & 19.0 \\
\hline 1951 & 1215 & 14.6 & 200.3 & 59.0 \\
\hline 1952 & 286 & 3.4 & 198.1 & 40.0 \\
\hline 1953 & 0 & 0.0 & 0.0 & 36.0 \\
\hline 1954 & 0 & 0.0 & 0.0 & 44.0 \\
\hline 1955 & 690 & 9.7 & 234.3 & 51.0 \\
\hline 1956 & 39 & 0.0 & 0.0 & 33.0 \\
\hline 1957 & 0 & 0.0 & 0.0 & 33.0 \\
\hline 1958 & 0 & 0.0 & 0.0 & 10.0 \\
\hline 1959 & 0 & 0.0 & 0.0 & 13.0 \\
\hline 1960 & 0 & 0.0 & 0.0 & 13.0 \\
\hline 1961 & 0 & 0.0 & 0.0 & 59.0 \\
\hline 1962 & 0 & 0.0 & 0.0 & 84.0 \\
\hline 1963 & 0 & 0.0 & 0.0 & 90.0 \\
\hline 1964 & 0 & 0.0 & 0.0 & 95.0 \\
\hline 1965 & 0 & 0.0 & 0.0 & 76.0 \\
\hline 1966 & 0 & 0.0 & 0.0 & 70.0 \\
\hline 1967 & 0 & 0.0 & 0.0 & 52.0 \\
\hline 1968 & 0 & 0.0 & 0.0 & 42.0 \\
\hline 1969 & 0 & 0.0 & 0.0 & 38.0 \\
\hline 1970 & 0 & 0.0 & 0.0 & 37.0 \\
\hline 1971 & 0 & 0.0 & 0.0 & 51.0 \\
\hline 1972 & 0 & 0.0 & 0.0 & 49.0 \\
\hline 1973 & 0 & 0.0 & 0.0 & 55.0 \\
\hline 1974 & 0 & 0.0 & 0.0 & 53.0 \\
\hline 1975 & 0 & 0.0 & 0.0 & 58.0 \\
\hline 1976 & 0 & 0.0 & 0.0 & 69.0 \\
\hline 1977 & 0 & 0.0 & 0.0 & 74.0 \\
\hline
\end{tabular}


WIIII. I.A-1H

\begin{tabular}{|c|c|c|c|c|c|c|c|}
\hline \multirow[b]{2}{*}{ Year } & \multirow[b]{2}{*}{$\begin{array}{c}\text { Pump Time } \\
\text { (h) }\end{array}$} & \multirow{2}{*}{$\begin{array}{c}\text { Pumpage } \\
\text { (million } \\
\text { gal) }\end{array}$} & \multirow[b]{2}{*}{$\begin{array}{l}\text { Pump Rate } \\
\text { (npm) }\end{array}$} & \multicolumn{2}{|c|}{ Water Ievel } & \multirow[b]{2}{*}{$\begin{array}{l}\text { Draw } \\
\text { Down }\end{array}$} & \multirow{2}{*}{$\begin{array}{l}\text { Specific } \\
\text { Capacity } \\
\text { (gpm/ft) }\end{array}$} \\
\hline & & & & $\begin{array}{l}\text { Non Pump } \\
\text { (fi) }\end{array}$ & $\begin{array}{c}\text { Pump } \\
\text { (ft) }\end{array}$ & & \\
\hline 19660 & 415 & 36.3 & 1457.8 & 3.0 & 131.0 & $3(1.4 .0$ & 14.19 \\
\hline$[96 j]$ & 3727 & 124.7 & 5.57 .6 & 54.9 & 154.1 & $10(0.0$ & $\therefore 6$ \\
\hline 19622 & .3936 & {$[29.1$} & 546.7 & 72.0 & 169.1 & 97.0 & 5.6 \\
\hline 1963 & 3649 & 117.4 & 5336.2 & 740 & 170.0 & 96.0 & 5.6 \\
\hline 1964 & 4174 & 130.3 & 520.3 & 81.0 & 18.3 .11 & 102.0 & 5.1 \\
\hline $196 \%$ & 30017 & 97.9 & 542.6 & 63.0 & 170.0 & 107.0 & 5.1 \\
\hline 19665 & 2589 & $8: 3.9$ & 540.1 & 50.0 & 169.0 & 119.11 & 4.5 \\
\hline 1967 & 2519 & 84.9 & 561.7 & 399.0 & 153.0 & 114.0 & 4.9 \\
\hline 1968 & $218: 3$ & 74.0 & 565.0 & 32.0 & 147.0 & 115.0 & 4.9 \\
\hline 1969 & 2244 & 75.7 & 562.2 & 22.1 & 142.0 & 120.0 & 4.7 \\
\hline 1970 & 2369 & 79.7 & 560.7 & 22.0 & 143.0 & 121.0 & 4.6 \\
\hline 1971 & 26333 & 89.1 & 564.0 & 31.0 & 162.0 & 131.0 & 4.3 \\
\hline 1972 & 2215 & 75.3 & 566.6 & 31.0 & 163.0 & 132.0 & 4.3 \\
\hline 1973 & 2628 & 87.2 & 55.3 .0 & 37.0 & 170.0 & 133.0 & 4.2 \\
\hline 1974 & 2282 & 73.9 & 539.7 & 35.0 & 161.0 & 126.0 & 4.3 \\
\hline 1975 & 2308 & 74.4 & 537.3 & 42.0 & 168.0 & 126.0 & 4.3 \\
\hline 1976 & 2521 & 79.6 & 526.2 & 50.0 & 176.0 & 126.0 & 4.2 \\
\hline 1977 & 2782 & 84.2 & 504.4 & 47.0 & 167.0 & 120.0 & 4.2 \\
\hline
\end{tabular}




\section{APPENISIX B (cont)}

WELI. I.A-2

\begin{tabular}{|c|c|c|c|c|c|c|c|}
\hline \multirow[b]{2}{*}{ Year } & \multirow[b]{2}{*}{$\begin{array}{c}\text { Pump Time } \\
\text { (h) }\end{array}$} & \multirow{2}{*}{$\begin{array}{c}\text { Pumpage } \\
\text { (million } \\
\text { gal) }\end{array}$} & \multirow[b]{2}{*}{$\begin{array}{l}\text { Pump Rute } \\
\text { (gpm) }\end{array}$} & \multicolumn{2}{|c|}{ Water Level } & \multirow[b]{2}{*}{$\begin{array}{l}\text { Jraw } \\
\text { Down }\end{array}$} & \multirow{2}{*}{$\begin{array}{l}\text { Specific } \\
\text { ('Apacity } \\
\text { (gpm/ft) }\end{array}$} \\
\hline & & & & $\begin{array}{c}\operatorname{Son} \text { Punip } \\
\text { (ft) }\end{array}$ & $\begin{array}{l}\text { Pump } \\
\text { (ft) }\end{array}$ & & \\
\hline 1947 & $4+1: 3$ & 27.6 & .97 .7 & $\ldots$ & & & .. \\
\hline $194-4$ & $365 i)^{4}$ & $59 . .3$ & 2.71 .1 & $\cdots$ & & -. & $\cdots$ \\
\hline 19.49 & $16 i \pi$ & $41 x$ & 421.2 & $\cdots$ & . & -. & $\cdots$ \\
\hline$\{45 ; 1\}$ & (i). & 15.6 & $42: 3 i$ & $.19 .1)$ & 24.511 & $2:-6.11$ & 1.9 \\
\hline $19: \overline{1}$ & 2415 & $5 \pi$ & $39 \times .2$ & 111.11 & $30 \div, 11$ & 19.4 .1 & 2.1 \\
\hline 1952 & $19 \times(1)$ & 46.18 & $3 \times 4.7$ & lili.1! & $3(11), 11$ & 199.11 & 2.0 \\
\hline 19523 & 22011 & 47.2 & $35 \pi .+4$ & 100.0 & 3011.11 & 2011.11 & 1.8 \\
\hline 1954 & $26(1)$ & $\overline{s i n}$ & $36-10$ & 116.11 & $\cdots$ & $\cdots$ & $\cdots$ \\
\hline $195 \overline{5}$ & 2,218 & 19.4 & 376.4 & 110.1 & $\cdots$ & $\cdots$ & $\cdots$ \\
\hline $19 i f_{i}$ & Imas & 4.4 .2 & $4(1) \times .1$ & 84.11 & - - & $\cdots$ & $\cdots$ \\
\hline $195 \%$ & $1116 f 5$ & 29.6 & 462.8 & 533.0 & 27.71 & 224.0 & 2.1 \\
\hline $195 \%$ & $11 \mathrm{fif}$ & $: 31.1$ & 444.5 & 60.01 & 2711.0 & 210.0 & 2.1 \\
\hline $19: 9$ & 1594 & 411.7 & 424.2 & 71.0 & $30: 3.0$ & $2: 320$ & 1.8 \\
\hline 19611 & 21694 & 51.6 & ד & 76.0 & $30 \div .0$ & 229.1 & 1.7 \\
\hline $196 i 1$ & 2149 & 44.4 & .344 .3 & 1011.0 & $31: 3.0$ & 212.11 & 1.6 \\
\hline 1962 & $182: 3$ & 35.7 & $: 326.4$ & 111.0 & 314.11 & 2013.0 & 1.6 \\
\hline $1965: 3$ & 1994 & $40 . \overline{7}$ & 339.3 & 127.6 & 3.32 .0 & $2(05.0)$ & 1.7 \\
\hline 1964 & 1924 & 34.2 & 296.3 & $1: 37.0$ & 347.0 & 210.0 & 1.4 \\
\hline 1965 & 1911 & 39.8 & 347.1 & 121.0 & 330.0 & $209.1)$ & 1.7 \\
\hline 1966 & 1070 & 21.4 & 333.3 & 108.0 & 340.0 & 232.0 & 1.4 \\
\hline 1967 & 238 & 4.9 & .343 .1 & 78.0 & 304.0 & 226.0 & 1.5 \\
\hline 1968 & 502 & 11.3 & 375.2 & 64.0 & 305.0 & 241.0 & 1.6 \\
\hline 1969 & $15 \overline{5}$ & 3.8 & 408.6 & 50.0 & 297.0 & 247.0 & $1 . \overline{7}$ \\
\hline 197() & 341 & 7.2 & 351.9 & 59.0 & 310.0 & 251.0 & 1.4 \\
\hline 1971 & 1787 & 31.8 & 296.6 & 88.0 & 318.0 & 230.0 & 1.3 \\
\hline 1972 & 2189 & 39.3 & 299.2 & 96.0 & 322.0 & 226.0 & 1.3 \\
\hline 1973 & 2625 & 46.7 & 296.5 & 106.0 & 334.0 & 228.0 & 1.3 \\
\hline 1974 & 203.3 & 36.8 & 301.7 & 109.0 & 325.0 & 216.0 & 1.4 \\
\hline 1975 & 2310 & 40.2 & 290.0 & 103.0 & 320.0 & 217.0 & 1.3 \\
\hline 1976 & 2488 & 39.9 & 267.3 & 113.0 & 322.0 & 209.0 & 1.3 \\
\hline $197 \%$ & 2775 & 42.5 & 255.3 & 118.0 & 314.0 & 196.0 & 1.3 \\
\hline
\end{tabular}


WHII, I.A-3

\begin{tabular}{|c|c|c|c|c|c|c|c|}
\hline \multirow[b]{2}{*}{ Year } & \multirow[b]{2}{*}{$\begin{array}{l}\text { Pump 'Time } \\
\text { (h) }\end{array}$} & \multirow{2}{*}{$\begin{array}{l}\text { Pumpage } \\
\text { (million } \\
\text { gal) }\end{array}$} & \multirow[b]{2}{*}{$\begin{array}{l}\text { Pump Rato } \\
\text { (gpm) }\end{array}$} & \multicolumn{2}{|c|}{ Water Ievel } & \multirow[b]{2}{*}{$\begin{array}{l}\text { Draw } \\
\text { Doun }\end{array}$} & \multirow{2}{*}{$\begin{array}{l}\text { Specific } \\
\text { Capacity } \\
\text { (gpm/ñt) }\end{array}$} \\
\hline & & & & $\begin{array}{c}\text { Son Pump } \\
\text { (fi) }\end{array}$ & $\begin{array}{l}\text { Pump } \\
\text { (fI) }\end{array}$ & & \\
\hline 19.7 & 1.76 & (i.4.9) & $732 x$ & $\cdots$ & $\cdots$ & $\cdots$ & $\cdots$ \\
\hline I9.1\% & 369.17 & $x: . . \overline{1}$ & :?ד.11 & $\cdots$ & $\cdots$ & $\ldots$ & . \\
\hline 1949 & $15(15)$ & 41.7 & $.16 i \mid x$ & $\cdots$ & $\cdots$ & $\cdots$ & $\cdots$ \\
\hline $19: 0$ & 2793 & $5 \pi x$ & 34.9 & 97.11 & $2: 31.11$ & 134.11 & $2.6 j$ \\
\hline $19: 1$ & sisit & $66 f .9$ & $31: 1.7$ & 116.0 & $2: 39.4$ & 117.11 & $2 . i$ \\
\hline 1952 & 251.1 & 58.6 & $38 x .5$ & 9.4 .0 & $2 \ln .1$ & 124.11 & 3.1 \\
\hline 19:is:3 & 3104 & 69.7 & 374.2 & 10.3 .0 & 2490 & $12 f i n$ & 3.0 \\
\hline 1954 & $2 \pi(4)$ & 57.3 & 365.11 & 101.0 & 225011 & 124.11 & 3.9 \\
\hline 1955 & 2195 & $4 \times .7$ & $369 . \times$ & 91.0 & $2 \cdot 26.11$ & 135.6 & 2.7 \\
\hline 1956 & 1849 & 42.1 & 379.5 & 74.0 & 222.0 & 148.11 & $\therefore .6$ \\
\hline 1957 & 1080 & 26.1 & 402.8 & 56.0 & 219.0 & 16.3 .11 & 2.5 \\
\hline 1958 & 1612 & 33.6 & 34.4 .4 & 49.0 & 225.0 & 176.0 & 2.0 \\
\hline 1959 & $18: 21$ & 35.0 & .320 .3 & 54.9 & 231.0 & 1770 & 1.8 \\
\hline 1960 & $2] 74$ & $3 \% .4$ & 294.4 & 68.0 & $2: 30.0$ & 162.0 & 1.8 \\
\hline 1961 & 1939 & 34.7 & 298.3 & 8.5 .0 & 189.0 & 104.0 & 2.9 \\
\hline 1962 & 2361 & 45.4 & $32(1.5$ & 93.0 & 192.0 & 99.0 & 3.2 \\
\hline 19633 & 2128 & 42.5 & 332.9 & 81.0 & 197.0 & 116.0 & 2.9 \\
\hline 1964 & 2574 & 50.4 & 326.3 & 104.0 & 217.0 & $11: 3.11$ & 2.9 \\
\hline 1965 & 1961 & 4.3 .4 & 368.9 & 79.0 & 220.0 & 141.0 & 2.6 \\
\hline 1966 & 2236 & 46.1 & 343.6 & 81.0 & 219.0 & 138.0 & 2.5 \\
\hline 1967 & 2274 & 47.4 & 347.4 & 86.0 & 218.0 & 132.0 & 2.6 \\
\hline 1968 & 2127 & 42.7 & 334.6 & 82.0 & 251.0 & 169.0 & 2.0 \\
\hline 1969 & 2072 & 40.1 & 322.6 & 58.0 & 246.0 & 188.0 & 1.7 \\
\hline 1970 & 2303 & 44.0 & 318.4 & 55.0 & 241.0 & 186.0 & 1.7 \\
\hline 1971 & 2556 & 45.4 & 296.0 & 77.0 & 250.0 & 173.0 & 1.7 \\
\hline 1972 & 2205 & 39.7 & 300.1 & 73.0 & 251.0 & 178.0 & 1.7 \\
\hline 1973 & 977 & 20.3 & 346.3 & 65.0 & 248.0 & 183.0 & 1.9 \\
\hline 1974 & 2291 & 43.5 & 316.5 & 73.0 & 244.0 & 171.0 & 1.9 \\
\hline 1975 & 2306 & 43.3 & 313.0 & 80.0 & 253.0 & 173.0 & 1.8 \\
\hline 1976 & 2474 & 42.3 & 285.0 & 88.0 & 260.0 & 172.0 & 1.7 \\
\hline 1977 & 2779 & 47.3 & 283.7 & 89.0 & 248.0 & 159.0 & 1.8 \\
\hline
\end{tabular}


WEIL. IA-1

\begin{tabular}{|c|c|c|c|c|c|c|c|}
\hline \multirow[b]{2}{*}{ Year } & \multirow[b]{2}{*}{$\begin{array}{c}\text { Pump Time } \\
\text { (h) }\end{array}$} & \multirow{2}{*}{$\begin{array}{c}\text { Pumpage } \\
\text { (million } \\
\text { gal) }\end{array}$} & \multirow[b]{2}{*}{$\begin{array}{c}\text { Pump Rate } \\
\text { (gpm) }\end{array}$} & \multicolumn{2}{|c|}{ Water level } & \multirow[b]{2}{*}{$\begin{array}{l}\text { Draw } \\
\text { Down }\end{array}$} & \multirow{2}{*}{$\begin{array}{l}\text { Specific } \\
\text { Capacity } \\
\text { (gpm/fi) }\end{array}$} \\
\hline & & & & $\begin{array}{c}\text { Non I'unip } \\
\text { (ft) }\end{array}$ & $\begin{array}{l}\text { Pump } \\
\text { (ft) }\end{array}$ & & \\
\hline $194 x$ & 1501 & 42.7 & $45,3.3$ & $\cdots$ & $\cdots$ & $\cdots$ & $\cdots$ \\
\hline 1949 & 940 & $37 . \overline{5}$ & 666.9 .9 & $\cdots$ & $\cdots$ & $\cdots$ & ... \\
\hline 1950$)$ & 4,350 & 164.9 & $0 ; 31 . x$ & $2-28.0$ & $: 3.3 .3 .0$ & $\overline{i .11}$ & $x .4$ \\
\hline 1951 & 4909 & 173.6 & $5 \times 9.4$ & $2 \times 5.0$ & 3.7 .011 & $7: 211$ & $x .2$ \\
\hline 1952 & $: 3429$ & 119.6 & $5 \times 1.3$ & 267.0 & $: 339.6$ & 7211 & 8.1 \\
\hline $195 \% 3$ & 30.34 & 109.1 & 599.8 & 26.4 .6 & $3: 5.6$ & 71.1 & 8.4 \\
\hline 195.4 & $21,3.3$ & 78.2 & 611.0 & 255.0 & $: 329.0$ & 7.4 .11 & $\times .3$ \\
\hline $195 \overline{5}$ & 2647 & 94.5 & 595.0 & 268.0 & 3.41 .0 & 73.11 & $\times .2$ \\
\hline 19565 & 3402 & 120.2 & 588.4 & 273.0 & 346.0 & 73.6 & 8.1 \\
\hline 1957 & 2844 & 105.4 & 617.7 & $27(1.0$ & 3.45 .0 & $7 \overline{1.11}$ & $x .2$ \\
\hline 1958 & 2973 & 110.3 & $61 \times .3$ & 270.0 & 342.0 & 72.11 & 8.6 \\
\hline 1959 & 3084 & 113.5 & 613.4 & 275.0 & 346.0 & 71.0 & 8.6 \\
\hline 1960 & 4084 & 145.6 & 594.2 & 296.0 & 365.0 & 69.0 & 8.6 \\
\hline 1961 & 3687 & 129.7 & 586.3 & 296.0 & 365.0 & 69.0 & 8.5 \\
\hline 1962 & 3688 & 129.3 & 584.3 & 286.0 & 359.0 & 73.0 & 8.0 \\
\hline 196.3 & 3718 & 130.5 & 585.0 & 280.0 & .351 .0 & 71.0 & 8.2 \\
\hline 1964 & 4500 & 155.0 & 574.1 & 291.0 & 361.0 & 70.0 & 8.2 \\
\hline 1965 & 3110 & 111.4 & 597.0 & 279.0 & 349.0 & 70.0 & 8.5 \\
\hline 1966 & 3279 & 115.6 & 587.6 & 285.0 & 356.0 & 71.0 & 8.3 \\
\hline 1967 & 2127 & 77.1 & 604.1 & 278.0 & 350.0 & 72.0 & 8.4 \\
\hline 1968 & 2276 & 81.7 & 598.3 & 280.0 & 351.0 & 71.0 & 8.4 \\
\hline 1969 & 1694 & 61.8 & 608.0 & 282.0 & 358.0 & 76.0 & 8.0 \\
\hline 1970 & 23.33 & 83.5 & 596.5 & 286.0 & 363.0 & 77.0 & 7.7 \\
\hline 1971 & 2519 & 89.0 & 588.9 & 287.0 & 373.0 & 86.0 & 6.8 \\
\hline 1972 & 2322 & 82.6 & 592.9 & 282.0 & 367.0 & 85.0 & 7.0 \\
\hline 1973 & 2616 & 92.4 & 588.7 & 294.0 & 377.0 & 83.0 & 7.1 \\
\hline 1974 & 2306 & 82.2 & 594.1 & 286.0 & 367.0 & 81.0 & 7.3 \\
\hline 1975 & 2319 & 82.3 & 591.5 & 272.0 & 355.0 & 83.0 & 7.1 \\
\hline 1976 & 2802 & 98.2 & 584.1 & 277.0 & 373.0 & 96.0 & 6.1 \\
\hline 1977 & 2741 & 96.4 & 586.2 & 278.0 & 374.0 & 96.0 & 6.1 \\
\hline
\end{tabular}


WEIL, LA-5)

\begin{tabular}{|c|c|c|c|c|c|c|c|}
\hline \multirow[b]{2}{*}{ Year } & \multirow[b]{2}{*}{$\begin{array}{c}\text { Pump Time } \\
\text { (h) }\end{array}$} & \multirow{2}{*}{$\begin{array}{c}\text { Pumpage } \\
\text { (million } \\
\text { gal) }\end{array}$} & \multirow[b]{2}{*}{$\begin{array}{c}\text { Pump Rate } \\
\text { (gpm) }\end{array}$} & \multicolumn{2}{|c|}{ Water Ievel } & \multirow[b]{2}{*}{$\begin{array}{l}\text { Draw } \\
\text { Down }\end{array}$} & \multirow{2}{*}{$\begin{array}{l}\text { Specific } \\
\text { Capacity } \\
\text { (gpm/ft) }\end{array}$} \\
\hline & & & & $\begin{array}{c}\text { Non Pump } \\
\text { (ft) }\end{array}$ & $\begin{array}{c}\text { Pump } \\
\text { (ft) }\end{array}$ & & \\
\hline 1948 & 1171 & 40.4 & 575.0 & $\cdots$ & $\ldots$ & $\ldots$ & $\ldots$ \\
\hline 1949 & 176.3 & 58.5 & $55: 3.0$ & -.- & --. & --. & $\ldots$ \\
\hline 1950 & $40 \div 2$ & 130.1 & 5335.1 & 131.0 & 254.0 & $12: 3.0$ & 4.4 \\
\hline 1951 & $600) 4$ & 187.4 & 520.2 & 162.0 & $2 \div 2.0$ & 110.9 & 4.7 \\
\hline 1952 & 3425 & 109.6 & 53.3 .3 & 147.0 & 259.0 & 112.0 & 4.8 \\
\hline $195: 3$ & 3278 & 103.9 & 528.3 & 141.0 & 257.0 & 116.0 & 4.6 \\
\hline 1954 & 2546 & 80.1 & 524.4 & 137.0 & 259.0 & 122.0 & 4.3 \\
\hline 1955 & 3158 & 97.3 & 513.5 & 145.0 & 267.0 & 122.0 & 4.2 \\
\hline 1956 & 3476 & 104.5 & 501.1 & 150.0 & 276.0 & 126.0 & 4.0 \\
\hline 1957 & 2868 & 86.0 & 499.8 & 150.0 & 277.0 & 127.0 & 3.9 \\
\hline 1958 & 3009 & 89.9 & 498.0 & 151.0 & 277.0 & 126.0 & 4.0 \\
\hline 1959 & 3088 & 93.5 & 504.6 & 15.5 .0 & 280.0 & 125.0 & 4.0 \\
\hline 1960 & 4088 & 119.1 & 485.6 & 168.0 & 288.0 & 120.0 & 4.0 \\
\hline 1961 & 35.34 & 100.3 & 473.0 & 165.0 & 288.0 & 123.6 & 3.8 \\
\hline 1962 & 3735 & 107.7 & 480.6 & 172.0 & -- & $\ldots$ & $\ldots$ \\
\hline 1963 & 3726 & 105.0 & 469.7 & 171.0 & -.- & $\ldots$ & ... \\
\hline 1964 & 4236 & 118.8 & 467.4 & 184.0 & $\cdots$ & $\ldots$ & $\cdots$ \\
\hline 1965 & 1740 & 50.5 & 483.7 & 180.0 & -- & -.- & -.- \\
\hline 1966 & 2817 & 79.3 & 469.2 & 180.0 & -- & $\ldots$ & $\ldots$ \\
\hline 1967 & 2533 & 73.7 & 484.9 & 168.0 & -.. & -- & --- \\
\hline 1968 & 2233 & 63.3 & 472.5 & 161.0 & 300.0 & 139.0 & 3.4 \\
\hline 1969 & 2402 & 68.5 & 475.3 & 161.0 & 298.0 & 137.0 & 3.5 \\
\hline 1970 & 2353 & 66.1 & 468.2 & 157.0 & 300.0 & 143.0 & 3.3 \\
\hline 1971 & 2659 & 74.4 & $\dot{4} 66.3$ & 155.0 & 302.0 & 147.0 & 3.2 \\
\hline 1972 & 2301 & 64.4 & 466.5 & 153.0 & 304.0 & 151.0 & 3.1 \\
\hline 1973 & 2476 & 68.3 & 459.7 & 156.0 & 308.0 & 152.0 & 3.0 \\
\hline 1974 & 1903 & 52.5 & 459.8 & 154.0 & 306.0 & 152.0 & 3.0 \\
\hline 1975 & 2318 & 63.9 & 459.4 & 149.0 & 309.0 & 160.0 & 2.9 \\
\hline 1976 & 2799 & 77.6 & 462.1 & 150.0 & 310.0 & 160.0 & 2.9 \\
\hline 1977 & 2665 & 74.8 & 467.8 & 147.0 & 303.0 & 156.0 & 3.0 \\
\hline
\end{tabular}




\section{APPENDIX B (cont)}

WELL IA-A

\begin{tabular}{|c|c|c|c|c|c|c|c|}
\hline \multirow[b]{2}{*}{ Year } & \multirow[b]{2}{*}{$\begin{array}{c}\text { Pump Time } \\
\text { (h) }\end{array}$} & \multirow{2}{*}{$\begin{array}{c}\text { Pumpage } \\
\text { (million } \\
\text { gal) } \\
\end{array}$} & \multirow[b]{2}{*}{$\begin{array}{c}\text { Pump Rate } \\
\text { (gpm) }\end{array}$} & \multicolumn{2}{|c|}{ Water level } & \multirow[b]{2}{*}{$\begin{array}{l}\text { Draw } \\
\text { Down }\end{array}$} & \multirow{2}{*}{$\begin{array}{l}\text { Specific } \\
\text { Capacity } \\
\text { (gpm/ft) }\end{array}$} \\
\hline & & & & $\begin{array}{l}\text { Non Pump } \\
(f t)\end{array}$ & $\begin{array}{c}\text { Pump } \\
\text { (ft) }\end{array}$ & & \\
\hline 1948 & 116 & 4.9 & $7(04.0$ & $\ldots$ & $\cdots$ & $\ldots$ & $\cdots$ \\
\hline 1949 & 2451 & 95.8 & 651.4 & $\ldots$ & -.. & $\ldots$ & $\ldots$ \\
\hline 1950 & 4490 & 167.9 & 623.2 & 8.3 .0 & $1: 36.0$ & 53.0 & 11.8 \\
\hline $19 \div 1$ & 5882 & 201.6 & 571.2 & $1 \mathrm{I} 5.0$ & 160.0 & 45.6 & 12.7 \\
\hline 1952 & 3168 & $110 . ?$ & 580.3 & 108.0 & 151.0 & 43.0 & 13.5 \\
\hline $195 \% 3$ & 3175 & 113.8 & 597.0 & 95.0 & 139.0 & 44.0 & 13.6 \\
\hline 1954 & 2894 & 107.1 & 616.8 & 92.0 & 135.0 & 43.0 & 14.3 \\
\hline $195 \overline{5}$ & 2911 & 108.0 & 618.3 & 97.0 & 140.0 & 43.0 & 14.4 \\
\hline 1956 & $34: 38$ & 125.8 & 609.9 & 106.0 & 149.0 & 43.0 & 14.2 \\
\hline 1957 & 28.3 .3 & 102.4 & 602.4 & 107.0 & 152.0 & 45.0 & 13.4 \\
\hline 1958 & 2957 & 106.9 & 602.5 & 108.0 & $1: 31.0$ & 43.0 & 14.0 \\
\hline 1959 & 3096 & 108.3 & 583.0 & 115.0 & 158.0 & 43.0 & 13.6 \\
\hline 1960 & 4084 & 138.6 & 565.6 & 130.0 & 172.0 & 42.0 & 13.5 \\
\hline 1961 & 3284 & 112.5 & 571.0 & 129.0 & 171.0 & 42.0 & 13.6 \\
\hline 1962 & 3886 & 129.4 & 555.0 & 135.0 & 175.0 & 40.0 & 13.9 \\
\hline $196: 3$ & 295.3 & 102.9 & 580.8 & 125.0 & 171.0 & 46.0 & 12.6 \\
\hline 1964 & 4244 & 138.3 & 543.1 & 132.0 & 172.0 & 40.0 & 13.6 \\
\hline 1965 & 3145 & 103.8 & 550.1 & 120.0 & 160.0 & 40.0 & 13.8 \\
\hline 1966 & 3173 & 104.0 & 546.3 & 129.0 & 169.0 & 40.0 & 13.7 \\
\hline 1967 & 2511 & 85.4 & 566.8 & 118.0 & 158.0 & 40.0 & 14.2 \\
\hline 1968 & 2111 & 71.6 & 565.3 & 109.0 & 150.0 & 41.0 & 13.8 \\
\hline 1969 & 2402 & 81.6 & 566.2 & 109.0 & 151.0 & 42.0 & 13.5 \\
\hline 1970 & 2337 & 79.1 & 564.1 & 106.0 & 149.0 & 43.0 & 13.1 \\
\hline 1971 & 2472 & 82.5 & 556.2 & 119.0 & 160.0 & 41.0 & 13.6 \\
\hline 1972 & 2317 & 79.2 & 569.7 & 117.0 & 155.0 & 38.0 & 15.0 \\
\hline 1973 & 2638 & 90.6 & 572.4 & 118.0 & 155.0 & 37.0 & 15.5 \\
\hline 1974 & 2337 & 79.8 & 569.1 & 120.0 & 156.0 & 36.0 & 15.8 \\
\hline 1975 & 1571 & 51.9 & 550.6 & 113.0 & 151.0 & 38.0 & 14.5 \\
\hline 1976 & 175 & 5.1 & 485.7 & 96.0 & -.- & --. & $\ldots$ \\
\hline 1977 & -.. &.- & ... & 82.0 & -- & ... & $\cdots$ \\
\hline
\end{tabular}


WEIL ( $;-1$

\begin{tabular}{|c|c|c|c|c|c|c|c|}
\hline \multirow[b]{2}{*}{ Year } & \multirow[b]{2}{*}{$\begin{array}{c}\text { Pump Time } \\
\text { (h) }\end{array}$} & \multirow{2}{*}{$\begin{array}{l}\text { Pumpage } \\
\text { (million } \\
\text { (gal) }\end{array}$} & \multirow[b]{2}{*}{$\begin{array}{l}\text { Pump Rate } \\
\text { (gpm) }\end{array}$} & \multicolumn{2}{|c|}{ Water level } & \multirow[b]{2}{*}{$\begin{array}{l}\text { Draw } \\
\text { Down }\end{array}$} & \multirow{2}{*}{$\begin{array}{l}\text { Specific } \\
\text { Capacit } \\
\text { ( } \mathrm{Fpm} / \mathrm{ft})\end{array}$} \\
\hline & & & & $\begin{array}{c}\text { Non Pump } \\
(f t)\end{array}$ & $\begin{array}{c}\text { Pump } \\
\text { (ft) }\end{array}$ & & \\
\hline 1950 & ( ) & 2.8 & 0.0 & 195.0 & $\ldots$ & $\ldots$ & $\ldots$ \\
\hline $19: 1$ & $116 x$ & 37.7 & $2 i 3.0$ & 202.0 & $: 309.0$ & 107.0 & 50 \\
\hline 1952 & 2476 & 75.5 & 5() 8.2 & 213.0 & 295.0 & 82.11 & 6.2 \\
\hline $19 ; 3$ & 3275 & 97.3 & 495.2 & 221.0 & 292.11 & 71.0 & 70 \\
\hline 1954 & 2616 & 77.8 & 495.7 & 221.0 & 290.10 & 69.11 & 7.2 \\
\hline 1955 & 24116 & 70.5 & 488.4 & 226.0 & 295.0 & 69.0 & 7.1 \\
\hline 1959 & 2958 & $8: 3.2$ & 468.8 & $2: 35.0$ & 303.3 & 68.0 & 6.9 \\
\hline $195 \overline{7}$ & 20.98 & 55.9 & 444.1 & 2.96 .1 & 307.0 & 71.0 & fi.3 \\
\hline 1958 & 2460 & 68.1 & 461.4 & $2: 38.0$ & 308.0 & 70.0 & 6.6 \\
\hline 1959 & 2952 & 82.4 & 465.2 & 245.0 & .314 .0 & 69.1 & 6.7 \\
\hline 1960 & 35664 & 96.0 & 448.9 & 254.0 & 325.0 & 71.0 & 6.3 \\
\hline 1961 & $42: 36$ & 112.4 & 442.2 & $26^{\prime} .0$ & 3333.0 & 73.0 & 6.1 \\
\hline 1962 & $: 34: 31$ & $9: 3.6$ & 454.7 & 258.0 & 342.0 & 84.0 & 5.4 \\
\hline 1963 & 4519 & 114.9 & 423.8 & 265.0 & 348.0 & 83.0 & 5.1 \\
\hline 1964 & $4: 374$ & 113.8 & 433.6 & 269.0 & 352.0 & 83.0 & 5.2 \\
\hline 1965 & 35330 & 90.7 & 428.2 & 268.0 & 352.0 & 84.0 & 5.1 \\
\hline 1966 & 4074 & 102.6 & 419.7 & 269.0 & 363.0 & 94.0 & 4.5 \\
\hline 1967 & 2615 & 69.9 & 445.5 & 266.0 & 362.0 & 96.0 & 4.6 \\
\hline 1968 & 2996 & 78.9 & 438.9 & 264.0 & 366.0 & 102.0 & 4.3 \\
\hline 1969 & 2657 & 68.3 & 428.4 & 266.0 & 376.0 & 110.0 & 3.9 \\
\hline 1970 & 2712 & 64.7 & 397.6 & 264.0 & 377.0 & 113.0 & 3.5 \\
\hline 1971 & 2908 & 67.9 & 389.2 & 258.0 & 378.0 & 120.0 & 3.2 \\
\hline 1972 & 2865 & 66.1 & 384.5 & 264.0 & 389.0 & 125.0 & 3.1 \\
\hline 1973 & 2997 & 67.5 & 375.4 & 271.0 & 403.0 & 132.0 & 2.8 \\
\hline 1974 & 2767 & 62.3 & 375.3 & 283.0 & 412.0 & 129.0 & 2.9 \\
\hline 1975 & 2467 & 55.7 & 376.3 & 293.0 & 411.0 & 118.0 & 3.2 \\
\hline 1976 & 2962 & 65.1 & 366.3 & -.. & -.. & -.- & $\ldots$ \\
\hline 1977 & 2734 & 57.9 & 353.0 & 275.0 & 426.0 & 151.0 & 2.3 \\
\hline
\end{tabular}




\section{APPENIDIX B (cont)}

WEI.I. (i-1A

\begin{tabular}{|c|c|c|c|c|c|c|c|}
\hline \multirow[b]{2}{*}{ Year } & \multirow[b]{2}{*}{$\begin{array}{c}\text { Pump Time } \\
\text { (h) }\end{array}$} & \multirow{2}{*}{$\begin{array}{c}\text { Pumpage } \\
\text { (million } \\
\text { gaI) }\end{array}$} & \multirow[b]{2}{*}{$\begin{array}{c}\text { Pump Rate } \\
\text { (gpm) } \\
\end{array}$} & \multicolumn{2}{|c|}{ Water Ievel } & \multirow[b]{2}{*}{$\begin{array}{l}\text { Draw } \\
\text { Down }\end{array}$} & \multirow{2}{*}{$\begin{array}{l}\text { Specific } \\
\text { Capacity } \\
(\mathrm{gpm} / \mathrm{ft})\end{array}$} \\
\hline & & & & $\begin{array}{l}\text { Non Pump } \\
\text { (ft) }\end{array}$ & $\begin{array}{c}\text { Pump } \\
\text { (ft) }\end{array}$ & & \\
\hline 1954 & 108 & 4.6 & 709.9 & -.- & .. & $\cdots$ & -.- \\
\hline 1955 & 1531 & $5,3.0$ & 577.0 & 265.0 & 316.0 & 51.1 & 11.3 \\
\hline 1956 & $31: 30$ & 107.7 & $57: 3.5$ & $27: 3.0$ & $32: 3.0$ & 50.0 & 11.5 \\
\hline $195 \overline{7}$ & 2470 & 87.0 & 587.0 & 274.0 & .327 .0 & 53.0 & 11.1 \\
\hline 1958 & 2670 & $\left.9^{\prime} ..\right)$ & 577.4 & 279.0 & $3: 31.0$ & 520 & $1 \mathrm{i} .1$ \\
\hline 1959 & 2965 & 102.7 & 577.3 & 284.0 & .333 .3 .0 & 49.0 & $11 . x$ \\
\hline 1960 & 3641 & 122.8 & 562.1 & 291.0 & .342 .0 & 51.0 & 11.0 \\
\hline 1961 & 4297 & 147.3 & 571.3 & 298.0 & 350.0 & 52.0 & 11.0 \\
\hline 1962 & .3972 & 1.36 .1 & 571.1 & 295.0 & 344.0 & 49.0 & $11 . \overline{7}$ \\
\hline 1963 & 4525 & 149.7 & 551.4 & 301.0 & 350.0 & 49.0 & 11.3 \\
\hline 1964 & .852 & 129.3 & 559.4 & 302.0 & 35.3 .0 & 51.0 & 11.0 \\
\hline 1965 & 3505 & 116.5 & 554.0 & 302.0 & 35.3 .0 & 51.0 & 10.9 \\
\hline 1966 & .3964 & 133.4 & 560.9 & 306.0 & 355.0 & 49.0 & 11.4 \\
\hline 1967 & 2720 & 91.3 & 559.4 & 302.0 & 351.0 & 49.0 & 11.4 \\
\hline 1968 & 3089 & 103.2 & $5 \tilde{6} 6.8$ & 302.0 & 352.0 & 50.0 & 11.1 \\
\hline 1969 & 2695 & 90.7 & 560.9 & 303.0 & 356.0 & 53.0 & 10.6 \\
\hline 1970 & 2772 & 92.5 & 556.2 & 300.0 & 357.0 & 57.0 & 9.8 \\
\hline 1971 & 3313 & 111.8 & 562.4 & 303.0 & 361.0 & 58.0 & 9.7 \\
\hline 1972 & 2879 & 94.0 & 544.2 & 302.0 & 361.0 & 59.0 & 9.2 \\
\hline 1973 & 2760 & 87.9 & 530.8 & 302.0 & 362.0 & 60.0 & 8.8 \\
\hline 1974 & 2974 & 92.7 & 519.5 & 307.0 & 355.0 & 48.0 & 10.8 \\
\hline 1975 & 2740 & 85.3 & 518.9 & 304.0 & 351.0 & 47.0 & 11.0 \\
\hline 1976 & 2983 & 91.6 & 511.8 & 302.0 & 350.0 & 48.0 & 10.7 \\
\hline 1977 & 2942 & 88.7 & 502.5 & 302.0 & 350.0 & 48.0 & 10.5 \\
\hline
\end{tabular}




\section{APPENIIX B (cont)}

WELL G-2

\begin{tabular}{|c|c|c|c|c|c|c|c|}
\hline \multirow[b]{2}{*}{ Year } & \multirow[b]{2}{*}{$\begin{array}{c}\text { Pump Time } \\
\text { (h) } \\
\end{array}$} & \multirow{2}{*}{$\begin{array}{c}\text { Pumpage } \\
\text { (million } \\
\text { gal) } \\
\end{array}$} & \multirow[b]{2}{*}{$\begin{array}{c}\text { Pump Rate } \\
\text { (gpm) }\end{array}$} & \multicolumn{2}{|c|}{ Water level } & \multirow[b]{2}{*}{$\begin{array}{l}\text { Draw } \\
\text { Down }\end{array}$} & \multirow{2}{*}{$\begin{array}{l}\text { Specific } \\
\text { Capacity } \\
\text { (gpm/ft) }\end{array}$} \\
\hline & & & & $\begin{array}{l}\text { Non Pump } \\
\text { (ft) }\end{array}$ & $\begin{array}{l}\text { Pump } \\
\text { (ft) }\end{array}$ & & \\
\hline 1951 & 123 & 3.9 & 528.5 & 259.0 & $\ldots$ & $\cdots$ & ... \\
\hline 1952 & 2.372 & 78.3 & 550.2 & 279.0 & 327.0 & 48.0 & 11.5 \\
\hline 195.3 & 3254 & 10.5 .6 & 540.9 & 290.0 & $3: 34.0$ & 44.0 & 12.3 \\
\hline 1954 & 2682 & 86.3 & 5.36 .3 & 291.0 & 335.0 & 44.1 & 12.2 \\
\hline 1955 & 2487 & 78.8 & 528.1 & 299.0 & 345.10 & 46.0 & 11.5 \\
\hline 1956 & 3109 & 95.8 & 513.6 & .310 .0 & 357.0 & 47.0 & 10.9 \\
\hline 1957 & 2458 & 76.1 & 516.0 & 311.0 & 360.0 & 49.1 & 111.5 \\
\hline 1958 & 2707 & 80.1 & 493.2 & 315.0 & 361.0 & 46.11 & 10.7 \\
\hline 1959 & 2938 & 84.6 & 479.9 & 320.0 & 36.3 .0 & 43.11 & 11.2 \\
\hline 1960 & 33535 & 96.6 & 455.4 & 328.0 & 370.0 & 42.1 & 10.8 \\
\hline 1961 & 3982 & 105.3 & 440.7 & $3: 36.0$ & 375.0 & 39.1 & 11.3 \\
\hline 1962 & 4076 & 99.8 & 408.1 & 338.0 & 374.0 & 36.0 & 11.3 \\
\hline 1963 & 4563 & 105.7 & 386.1 & 344.0 & 379.0 & 35.0 & 11.0 \\
\hline 1964 & 4541 & 105.3 & 386.5 & 346.0 & 380.0 & 34.0 & 11.4 \\
\hline 1965 & 35.35 & 82.6 & 389.4 & 346.0 & 381.0 & 35.0 & 11.1 \\
\hline 1966 & 3994 & 94.7 & 395.2 & 349.0 & 38.3 .0 & 34.0 & 11.6 \\
\hline 1967 & 2743 & 67.6 & 410.7 & 344.0 & 379.0 & 35.0 & 11.7 \\
\hline 1968 & 2732 & 66.5 & 405.7 & 344.0 & 379.0 & 35.0 & 11.6 \\
\hline 1969 & 2679 & 68.6 & 426.8 & 344.0 & 381.0 & 37.0 & 11.5 \\
\hline 1970 & 2431 & 62.8 & 430.5 & 343.0 & 381.0 & 38.0 & 11.3 \\
\hline 1971 & 3420 & 87.4 & 425.9 & 345.0 & 384.0 & 39.0 & 10.9 \\
\hline 1972 & 2897 & 73.4 & 423.7 & 348.0 & 388.0 & 40.0 & 10.6 \\
\hline 1973 & 2816 & 72.4 & 428.5 & 344.0 & 385.0 & 41.0 & $10 . \tilde{5}$ \\
\hline 1974 & 3056 & 82.0 & 447.2 & 347.0 & 390.0 & 43.0 & 10.4 \\
\hline 1975 & 2724 & 74.5 & 455.8 & 341.0 & 384.0 & 43.0 & 10.6 \\
\hline 1976 & 2990 & 81.1 & 452.1 & 344.0 & 388.0 & 44.0 & 10.3 \\
\hline 1977 & 2981 & 80.4 & 449.5 & 346.0 & 388.0 & 42.0 & $10 . \bar{T}$ \\
\hline
\end{tabular}




\section{APPENDIX B (cont)}

WELL G-3

\begin{tabular}{|c|c|c|c|c|c|c|c|}
\hline \multirow[b]{2}{*}{ Year } & \multirow[b]{2}{*}{$\begin{array}{c}\text { Pump Time } \\
\text { (h) } \\
\end{array}$} & \multirow{2}{*}{$\begin{array}{c}\text { Pumpage } \\
\text { (million } \\
\text { gal) } \\
\end{array}$} & \multirow[b]{2}{*}{$\begin{array}{c}\text { Pump Rate } \\
\text { (gpm) }\end{array}$} & \multicolumn{2}{|c|}{ Water Level } & \multirow[b]{2}{*}{$\begin{array}{l}\text { Draw } \\
\text { Down }\end{array}$} & \multirow{2}{*}{$\begin{array}{l}\text { Specific } \\
\text { Capacity } \\
\text { (gpm/nt) }\end{array}$} \\
\hline & & & & $\begin{array}{c}\begin{array}{c}\text { Non Pump } \\
\text { (ft) }\end{array} \\
\end{array}$ & $\begin{array}{c}\text { Pump } \\
\text { (ft) }\end{array}$ & & \\
\hline 1951 & 192 & 7.3 & 633.7 & 281.0 & $\ldots$ & $\cdots$ & $\ldots$ \\
\hline 1952 & 2379 & 65.4 & 458.2 & 310.0 & 358.0 & 48.0 & 9.5 \\
\hline 195.3 & 3192 & 76.4 & 398.9 & 322.0 & 360.0 & 38.0 & 10.5 \\
\hline 1954 & 2675 & 66.1 & 411.8 & 322.0 & 370.0 & 48.0 & 8.6 \\
\hline 195.5 & $2: 369$ & 69.4 & 488.3 & 316.0 & 368.0 & 52.0 & 9.4 \\
\hline 1956 & $: 3149$ & 87.9 & 465.2 & 324.0 & 380.0 & 56.0 & 8.3 \\
\hline $195 \%$ & 2517 & 70.2 & 464.8 & 324.0 & 385.0 & 61.0 & 7.6 \\
\hline 1958 & 2562 & 69.5 & 452.1 & 323.0 & 386.0 & 63.0 & 7.2 \\
\hline 1959 & $29: 31$ & 74.6 & 424.2 & 326.0 & 395.0 & 69.0 & 6.1 \\
\hline 1960 & 3591 & 82.5 & 382.9 & 335.0 & 407.0 & 72.0 & 5.3 \\
\hline 1961 & 3612 & 79.9 & 368.7 & 343.0 & 414.0 & 71.0 & 5.2 \\
\hline 1962 & 4057 & 83.7 & 343.9 & 348.0 & 418.0 & 70.0 & 4.9 \\
\hline 1963 & 4555 & 86.7 & 317.2 & 352.0 & 422.0 & 70.0 & 4.5 \\
\hline 1964 & 4487 & 78.6 & 292.0 & 355.0 & 424.0 & 69.0 & 4.2 \\
\hline 1965 & 3498 & 65.6 & 312.6 & 350.0 & 419.0 & 69.0 & 4.5 \\
\hline 1966 & 3991 & 73.7 & 307.8 & 353.0 & 420.0 & 67.0 & 4.6 \\
\hline 1967 & 2752 & 52.9 & 320.4 & 344.0 & 418.0 & 74.0 & 4.3 \\
\hline 1968 & 3086 & 56.5 & 305.1 & 341.0 & 418.0 & 77.0 & 4.0 \\
\hline 1969 & 2672 & 50.8 & 316.9 & 338.0 & 417.0 & 79.0 & 4.0 \\
\hline 1970 & 2736 & 55.4 & 337.5 & 336.0 & 419.0 & 83.0 & 4.1 \\
\hline 1971 & 3337 & 64.2 & 320.6 & 342.0 & 423.0 & 81.0 & 4.0 \\
\hline 1972 & 2838 & 50.9 & 298.9 & 341.0 & 421.0 & 80.0 & 3.7 \\
\hline 1973 & 2843 & 47.3 & 277.3 & 341.0 & 418.0 & 77.0 & 3.6 \\
\hline 1974 & 3006 & 49.3 & 273.3 & 342.0 & 424.0 & 82.0 & 3.3 \\
\hline 1975 & 2632 & 43.1 & 272.9 & 341.0 & 428.0 & 87.0 & 3.1 \\
\hline 1976 & 2971 & 82.6 & 463.4 & 374.0 & 462.0 & 88.0 & 5.3 \\
\hline 1977 & 2961 & 78.9 & 444.1 & 368.0 & 463.0 & 95.0 & 4.7 \\
\hline
\end{tabular}


WELI, ( $\mathrm{i-1}$

\begin{tabular}{|c|c|c|c|c|c|c|c|}
\hline \multirow[b]{2}{*}{ Year } & \multirow[b]{2}{*}{$\begin{array}{c}\text { Pump Time } \\
\text { (h) }\end{array}$} & \multirow{2}{*}{$\begin{array}{c}\text { Pumpage } \\
\text { (million } \\
\text { (gal) }\end{array}$} & \multirow[b]{2}{*}{$\begin{array}{c}\text { Pump Rate } \\
\text { (gpm) }\end{array}$} & \multicolumn{2}{|c|}{ Water Level } & \multirow[b]{2}{*}{$\begin{array}{l}\text { Draw } \\
\text { Down }\end{array}$} & \multirow{2}{*}{$\begin{array}{l}\text { Specific } \\
\text { Capacity } \\
\text { (gpm/ft) }\end{array}$} \\
\hline & & & & $\begin{array}{c}\text { Non Pump } \\
\text { (ft) }\end{array}$ & $\begin{array}{c}\text { Pump } \\
\text { (ft) }\end{array}$ & & \\
\hline 1951 & -.. & 12.5 & ..- & 357.0 & 477.0 & 120.0 &.- \\
\hline 1952 & 2401 & 56.9 & 395.0 & 374.0 & 474.0 & 100.0 & 3.9 \\
\hline $195: 3$ & 2677 & 55.2 & $34: 3.7$ & 380.0 & 472.0 & 920 & 3.7 \\
\hline 1954 & 2256 & 58.8 & 434.4 & 383.0 & 526.0 & 143.0 & 3.0 \\
\hline 1955 & 1172 & 22.7 & 322.8 & 378.0 & 481.0 & 10.3 .0 & 3.1 \\
\hline 1956 & 1800 & 33.9 & 313.9 & 377.0 & 491.0 & 114.0 & 2.8 \\
\hline 1957 & $1: 324$ & 24.2 & 304.6 & 373.0 & 498.0 & 125.0 & 2.4 \\
\hline 1958 & 1970 & 35.9 & 303.7 & 370.0 & 490.0 & 120.0 & 2.5 \\
\hline 1959 & 1819 & 31.6 & $289 . \overline{5}$ & 378.1 & 494.1 & 116.0 & 2.5 \\
\hline 1960 & 2457 & 37.0 & 251.0 & 385.0 & $5(19.0$ & 124.0 & 2.0 \\
\hline 1961 & 2787 & 45.0 & 269.1 & 389.0 & 512.0 & 123.0 & 2.2 \\
\hline 19632 & $27: 38$ & 41.7 & 25.3 .8 & 386.0 & 505.0 & 119.0 & 2.1 \\
\hline 1963 & 3519 & 46.4 & 219.8 & 388.0 & 504.0 & 116.0 & 1.9 \\
\hline 1964 & 35661 & 42.9 & 200.8 & 396.0 & 499.0 & 103.0 & 1.9 \\
\hline 1965 & 2100 & 23.8 & 188.9 & 894.0 & 492.0 & 98.0 & 1.9 \\
\hline 1966 & 2219 & 33.6 & 252.4 & 391.0 & 498.0 & 107.0 & 2.4 \\
\hline 1967 & 2690 & 44.8 & 277.6 & 388.0 & 509.0 & 121.0 & 2.3 \\
\hline 1968 & 208.3 & 31.4 & 251.2 & 386.0 & 509.0 & 123.0 & 2.0 \\
\hline 1969 & 1309 & 17.4 & 221.5 & 387.0 & 505.0 & 118.0 & 1.9 \\
\hline 1970 & 606 & 7.7 & 211.8 & 384.0 & 504.0 & 120.0 & 1.8 \\
\hline 1971 & 1640 & 21.0 & 21.3 .4 & 389.0 & 503.0 & 114.0 & 1.9 \\
\hline 1972 & 2840 & 33.3 & 195.4 & 391.0 & 507.0 & 116.0 & 1.7 \\
\hline 1973 & 3006 & 37.2 & 206.3 & 392.0 & 521.0 & 129.0 & 1.6 \\
\hline 1974 & 2672 & 34.3 & 213.9 & 392.0 & 519.0 & 127.0 & 1.7 \\
\hline 1975 & 1977 & 41.0 & 345.6 & 403.0 & 559.0 & 156.0 & 2.2 \\
\hline 1976 & 2859 & 57.8 & 336.9 & 406.0 & 571.0 & 165.0 & 2.0 \\
\hline 1977 & 2954 & 62.4 & 352.1 & 406.0 & 589.0 & 183.0 & 1.9 \\
\hline
\end{tabular}


WELL G-5

\begin{tabular}{|c|c|c|c|c|c|c|c|}
\hline \multirow[b]{2}{*}{ Year } & \multirow[b]{2}{*}{$\begin{array}{c}\text { Pump Time } \\
\text { (h) }\end{array}$} & \multirow{2}{*}{$\begin{array}{c}\text { Pumpage } \\
\text { (million } \\
\text { gal) } \\
\end{array}$} & \multirow[b]{2}{*}{$\begin{array}{c}\text { Pump Rate } \\
\text { (gpm) }\end{array}$} & \multicolumn{2}{|c|}{ Water Level } & \multirow[b]{2}{*}{$\begin{array}{l}\text { Draw } \\
\text { Down }\end{array}$} & \multirow{2}{*}{$\begin{array}{l}\text { Specific } \\
\text { Capacity } \\
\text { (gpm/ft) }\end{array}$} \\
\hline & & & & $\begin{array}{c}\text { Non Pump } \\
\text { (ft) } \\
\end{array}$ & $\begin{array}{c}\text { Pump } \\
\text { (ft) }\end{array}$ & & \\
\hline 1951 & --- & 6.7 & -.- & 414.0 & ... & -- &.-- \\
\hline 1952 & 2579 & 73.8 & 476.9 & 422.0 & 480.0 & 58.0 & 8.2 \\
\hline 1953 & 1433 & 37.8 & 439.6 & 425.0 & 467.0 & 42.0 & 10.5 \\
\hline 1954 & 2617 & 80.9 & 515.2 & 429.9 & 473.0 & 44.0 & $11 . \overline{1}$ \\
\hline 1955 & 2529 & 80.4 & 529.9 & 427.0 & 472.0 & 45.0 & 11.8 \\
\hline 1956 & 3052 & 97.0 & 529.7 & 4.31 .0 & 478.0 & 47.0 & 11.3 \\
\hline 1957 & 2385 & 64.1 & 447.9 & 424.0 & 466.0 & 42.0 & $10 . \overline{7}$ \\
\hline 1958 & 1523 & 49.1 & 5.37 .3 & 428.0 & 477.0 & 49.0 & 11.0 \\
\hline 1959 & 2917 & 101.7 & 581.1 & 435.0 & 495.0 & 60.0 & 9.7 \\
\hline 1960 & 2828 & 98.0 & 577.6 & 437.0 & 501.0 & 64.0 & 9.0 \\
\hline 1961 & 3908 & 134.0 & 571.5 & 438.0 & 507.0 & 69.0 & 8.3 \\
\hline 1962 & 4186 & 142.0 & 565.4 & 440.0 & 511.0 & 71.0 & 8.0 \\
\hline 1963 & 4528 & 151.0 & 555.8 & 441.0 & 513.0 & 72.0 & 7.7 \\
\hline 1964 & 4532 & 150.4 & 553.1 & 446.0 & 516.0 & 70.0 & 7.9 \\
\hline 1965 & 3520 & 117.1 & 554.5 & 443.0 & 516.0 & 73.0 & 7.6 \\
\hline 1966 & 2555 & 83.2 & 542.7 & 445.0 & 520.0 & 75.0 & 7.2 \\
\hline 1967 & 2405 & 80.0 & 554.4 & 444.0 & 519.0 & 75.0 & 7.4 \\
\hline 1968 & 2513 & 81.2 & 538.5 & 443.0 & 517.0 & 74.0 & 7.3 \\
\hline 1969 & 2649 & 83.3 & 524.1 & 450.0 & 520.0 & 70.0 & 7.5 \\
\hline 1970 & 2771 & 88.9 & 534.7 & 453.0 & $\overline{5} 21.0$ & 68.0 & 7.9 \\
\hline 1971 & 2657 & 88.3 & 553.9 & 450.0 & 521.0 & 71.0 & 7.8 \\
\hline 1972 & 2902 & 92.4 & 530.7 & 441.0 & 514.0 & 73.0 & 7.3 \\
\hline 1973 & 3003 & 97.5 & 541.1 & 444.0 & 515.0 & 71.0 & 7.6 \\
\hline $197:$ & 2054 & 69.0 & 559.9 & 440.0 & 513.0 & 73.0 & 7.7 \\
\hline 1975 & 2266 & 74.7 & 549.4 & 433.0 & 500.0 & 67.0 & 8.2 \\
\hline 1976 & 2955 & 95.0 & 535.8 & 442.0 & 504.0 & 62.0 & 8.6 \\
\hline 1977 & 2836 & 92.1 & 541.3 & 444.0 & 504.0 & 60.0 & 9.0 \\
\hline
\end{tabular}




\section{APPENDIX B (cont)}

WELL G-6

\begin{tabular}{|c|c|c|c|c|c|c|c|}
\hline \multirow[b]{2}{*}{ Year } & \multirow[b]{2}{*}{$\begin{array}{c}\text { Pump Time } \\
\text { (h) }\end{array}$} & \multirow{2}{*}{$\begin{array}{c}\text { Pumpage } \\
\text { (million } \\
\text { gal) }\end{array}$} & \multirow[b]{2}{*}{$\begin{array}{c}\text { Pump Rate } \\
\text { (gpm) }\end{array}$} & \multicolumn{2}{|c|}{ Water Level } & \multirow[b]{2}{*}{$\begin{array}{l}\text { Draw } \\
\text { Down }\end{array}$} & \multirow{2}{*}{$\begin{array}{l}\text { Specific } \\
\text { Capacity } \\
\text { (gpm/ft) }\end{array}$} \\
\hline & & & & $\begin{array}{c}\text { Non Pump } \\
\text { (ft) }\end{array}$ & $\begin{array}{c}\text { Pump } \\
\text { (ft) }\end{array}$ & & \\
\hline 1964 & 1912 & 45.0 & 392.3 & $58 i 0$ & 659.0 & 78.0 & 5.0 \\
\hline 1965 & 3200 & 74.9 & 390.1 & 582.0 & 660.0 & 78.0 & 5.0 \\
\hline 1966 & 3931 & 92.2 & 390.9 & 585.0 & 658.0 & 73.0 & 5.4 \\
\hline 1967 & 2454 & 57.8 & 392.6 & 580.0 & 653.0 & 73.0 & 5.4 \\
\hline 1968 & 2597 & 56.2 & 360.7 & 574.0 & 647.0 & 73.0 & 4.9 \\
\hline 1969 & 2698 & 55.6 & 343.5 & 568.0 & 636.0 & 68.0 & 5.1 \\
\hline 1970 & 2765 & 51.0 & 307.4 & 569.0 & 634.0 & 65.0 & 4.7 \\
\hline 1971 & 2932 & 42.8 & 243.3 & 573.0 & 629.0 & 56.0 & 4.3 \\
\hline 1972 & 2516 & 57.0 & 377.6 & 578.0 & 670.0 & 92.0 & 4.1 \\
\hline 1973 & 2991 & 65.3 & 363.9 & 579.0 & 667.0 & 88.0 . & 4.1 \\
\hline 1974 & 2950 & 63.8 & 360.5 & 579.0 & 665.0 & 86.0 & 4.2 \\
\hline 1975 & 2717 & 56.7 & 347.8 & 577.0 & 659.0 & 82.0 & 4.2 \\
\hline 1976 & 2966 & 57.8 & 324.8 & 584.0 & 662.0 & 78.0 & 4.2 \\
\hline 1977 & 2954 & 54.4 & 306.9 & 586.0 & 659.0 & 73.0 & 4.2 \\
\hline
\end{tabular}

WELL PM-1

\begin{tabular}{|c|c|c|c|c|c|c|c|}
\hline \multirow[b]{2}{*}{ Year } & \multirow[b]{2}{*}{$\begin{array}{c}\text { Pump Time } \\
\text { (h) }\end{array}$} & \multirow{2}{*}{$\begin{array}{c}\text { Pumpage } \\
\text { (million } \\
\text { gal) } \\
\end{array}$} & \multirow[b]{2}{*}{$\begin{array}{c}\text { Pump Rate } \\
\text { (gpm) }\end{array}$} & \multicolumn{2}{|c|}{ Water Level } & \multirow[b]{2}{*}{$\begin{array}{l}\text { Draw } \\
\text { Down }\end{array}$} & \multirow{2}{*}{$\begin{array}{l}\text { Specific } \\
\text { Capacity } \\
\text { (gpm/ft) }\end{array}$} \\
\hline & & & & $\begin{array}{c}\text { Non Pump } \\
\text { (ft) }\end{array}$ & $\begin{array}{c}\text { Pump } \\
\mathbf{f t})\end{array}$ & & \\
\hline 1965 & 2754 & 99.2 & 600.3 & 746.0 & 786.0 & 40.0 & 15.0 \\
\hline 1966 & 3086 & 108.0 & 583.3 & 740.0 & 779.0 & 39.0 & 15.0 \\
\hline 1967 & 2870 & 111.0 & 644.6 & 737.0 & 781.0 & 44.0 & 14.6 \\
\hline 1968 & 1846 & 68.1 & 614.8 & 735.0 & 769.0 & 34.0 & 18.1 \\
\hline 1969 & 951 & 34.4 & 602.9 & 733.0 & 766.0 & 33.0 & 18.3 \\
\hline 1970 & 1781 & 66.2 & 619.5 & 733.0 & 769.0 & $\$ 6.0$ & 17.2 \\
\hline 1971 & 2728 & 101.0 & 617.1 & 733.0 & 766.0 & 33.0 & 18.7 \\
\hline 1972 & 2415 & 84.9 & 585.9 & 735.0 & 762.0 & 27.0 & 21.7 \\
\hline 1973 & 1688 & 46.5 & 459.1 & 736.0 & 755.0 & 19.0 & 24.2 \\
\hline 1974 & 2649 & 96.3 & 605.9 & 740.0 & 768.0 & 28.0 & 21.6 \\
\hline 1975 & 2567 & 94.8 & 615.5 & 741.0 & 766.0 & 25.0 & 24.6 \\
\hline 1976 & 2933 & 106.8 & 606.9 & 744.0 & 767.0 & 23.0 & 26.4 \\
\hline 1977 & 2969 & 105.4 & 591.7 & 745.0 & 767.0 & 22.0 & 26.9 \\
\hline
\end{tabular}


APPENDIX B (cont)

WELL PM-2

\begin{tabular}{|c|c|c|c|c|c|c|c|}
\hline \multirow[b]{2}{*}{ Year } & \multirow[b]{2}{*}{$\begin{array}{c}\text { Pump Time } \\
\text { (h) }\end{array}$} & \multirow{2}{*}{$\begin{array}{c}\text { Pumpage } \\
\text { (million } \\
\text { gal) } \\
\end{array}$} & \multirow[b]{2}{*}{$\begin{array}{c}\text { Pump Rate } \\
\text { (gpm) }\end{array}$} & \multicolumn{2}{|c|}{ Water Level } & \multirow[b]{2}{*}{$\begin{array}{l}\text { Draw } \\
\text { Down }\end{array}$} & \multirow{2}{*}{$\begin{array}{c}\text { Specific } \\
\text { Capacity } \\
\text { (gpm/ft) }\end{array}$} \\
\hline & & & & $\begin{array}{l}\text { Non Pump } \\
\text { (ft) }\end{array}$ & $\begin{array}{l}\text { Pump } \\
\text { (ft) }\end{array}$ & & \\
\hline 1966 & 221 & 18.9 & 1425.3 & 826.0 & 889.0 & j.3.0 & 22.6 \\
\hline 1967 & 4336 & 370.0 & 1422.2 & 834.0 & 888.0 & 54.0 & 26.3 \\
\hline 1968 & 3865 & 328.2 & 1415.3 & 838.0 & 889.0 & 51.0 & 27.8 \\
\hline 1969 & 3304 & 279.9 & 1411.9 & 838.0 & 890.0 & 52.0 & 27.2 \\
\hline 1970 & 3529 & 300.6 & 1419.7 & 839.0 & 893.0 & 54.0 & 26.3 \\
\hline 1971 & 4035 & 339.5 & 1402.3 & 841.0 & 898.0 & 57.0 & 24.6 \\
\hline 1972 & 4611 & 385.3 & 1392.7 & 845.0 & 902.0 & 57.0 & 24.4 \\
\hline 1973 & 4571 & 380.6 & 1387.7 & 849.0 & 907.0 & 58.0 & 23.9 \\
\hline 1974 & 5443 & 450.9 & 1380.7 & 853.0 & 912.0 & 59.0 & 23.4 \\
\hline 1975 & 4644 & 385.3 & 1382.8 & 854.0 & 913.0 & 59.0 & 23.4 \\
\hline 1976 & 5382 & 442.0 & 1368.8 & 866.0 & 924.0 & 58.0 & 23.6 \\
\hline 1977 & 3306 & 272.8 & 1375.3 & 868.0 & 924.0 & 56.0 & 24.6 \\
\hline
\end{tabular}

WELL PM-3

\begin{tabular}{|c|c|c|c|c|c|c|c|}
\hline \multirow[b]{2}{*}{ Year } & \multirow[b]{2}{*}{$\begin{array}{c}\text { Pump Time } \\
\text { (h) }\end{array}$} & \multirow{2}{*}{$\begin{array}{c}\text { Pumpage } \\
\text { (million } \\
\text { gal) }\end{array}$} & \multirow[b]{2}{*}{$\begin{array}{c}\text { F . Imp Rate } \\
\text { (gpm) }\end{array}$} & \multicolumn{2}{|c|}{ Water Level } & \multirow[b]{2}{*}{$\begin{array}{l}\text { Draw } \\
\text { Down }\end{array}$} & \multirow{2}{*}{$\begin{array}{l}\text { Specific } \\
\text { Capacity } \\
\text { (gpm/ft) }\end{array}$} \\
\hline & & & & $\begin{array}{c}\text { Non Pump } \\
\text { (ft) }\end{array}$ & $\begin{array}{c}\text { Pump } \\
\text { (ft) }\end{array}$ & & \\
\hline 1968 & 2327 & 187.4 & 1342.2 & 743.0 & 771.0 & 28.0 & 47.9 \\
\hline 1969 & 3241 & 254.7 & 1309.8 & 746.0 & 772.0 & $26 . t^{\prime}$ & 50.4 \\
\hline 1970 & 2905 & 227.8 & 1306.9 & 750.0 & 774.0 & 24.6 & 54.5 \\
\hline 1971 & 2774 & 216.3 & 1299.6 & 751.0 & 774.0 & 23.0 & 56.5 \\
\hline 1972 & 2445 & 192.1 & 1309.5 & 752.0 & 775.0 & 23.0 & 56.9 \\
\hline 1973 & 3256 & 257.8 & 1319.6 & 755.0 & 778.0 & 23.0 & 57.4 \\
\hline 1974 & 3241 & 255.3 & 1312.9 & 756.0 & 779.0 & 23.0 & 57.1 \\
\hline 1975 & 3421 & 269.3 & 1312.0 & 757.0 & 780.0 & 23.0 & 57.0 \\
\hline $1 \mathrm{c} 76$ & 3171 & 268.3 & 1410.2 & 758.0 & 784.0 & 26.0 & 54.2 \\
\hline $19 \%$ & 2792 & 235.5 & 1405.8 & 758.0 & 784.0 & 26.0 & 54.1 \\
\hline
\end{tabular}


WATER CANYON GALLERY

\begin{tabular}{|c|c|c|c|}
\hline Yeur & $\begin{array}{c}\text { Pump Time } \\
\text { (h) }\end{array}$ & $\begin{array}{c}\text { Pumpage } \\
\text { (million } \\
\text { gal) }\end{array}$ & $\begin{array}{c}\text { Rate } \\
\text { (gpm) }\end{array}$ \\
\hline 1947 & 8760 & 84.0 & 159.8 \\
\hline 1948 & 8784 & 97.0 & 184.0 \\
\hline 1949 & 8760 & 92.0 & 175.0 \\
\hline 1950 & 8760 & 54.0 & 102.7 \\
\hline $19 \overline{1} 1$ & 8760 & 39.0 & 74.2 \\
\hline 195,2 & 8784 & $48.1)$ & 91.1 \\
\hline 195.1 & 8760 & 39.0 & 74.2 \\
\hline 1954 & 8760 & 40.0 & 76.1 \\
\hline 195.5 & 8760 & 33.0 & 62.8 \\
\hline 1956 & 8784 & 23.0 & 43.6 \\
\hline 1957 & 8760 & 40.0 & 76.1 \\
\hline 1958 & 8760 & 60.0 & 114.2 \\
\hline 1959 & 8760 & 54.0 & 102.7 \\
\hline 1960 & 8784 & 48.0 & 91.1 \\
\hline 1961 & 8760 & 54.0 & 102.7 \\
\hline 1962 & 8760 & 67.0 & 127.5 \\
\hline 1963 & 8760 & 51.0 & 97.0 \\
\hline 1964 & 8784 & 45.0 & 85.4 \\
\hline 1965 & 8760 & 72.0 & 137.0 \\
\hline 1966 & 8760 & 82.0 & 156.0 \\
\hline 1967 & 8760 & 56.0 & 106.5 \\
\hline 1968 & 8784 & 65.0 & 123.3 \\
\hline 1.969 & 8760 & 80.0 & 152.2 \\
\hline 1970 & 8760 & 65.0 & 123.7 \\
\hline 1971 & 8760 & 37.0 & 70.4 \\
\hline 1972 & 8784 & 40.0 & 75.9 \\
\hline 1973 & 8760 & 49.0 & 93.2 \\
\hline 1974 & 8760 & 35.0 & 66.6 \\
\hline 1975 & 8760 & 42.0 & 79.9 \\
\hline 1976 & 8784 & 41.0 & 77.8 \\
\hline 1977 & 8760 & 57.0 & 108.4 \\
\hline
\end{tabular}

